\title{
Construction of the MEGA photon detector ${ }^{+}$
}

\author{
M. Barakat ${ }^{\text {a,i }}$, Y. Chen ${ }^{a}$, S. Conrad ${ }^{\text {a,b }}$, M. Cooper ${ }^{\text {c }}$, M. Dzemidzic ${ }^{\text {a }}$, J.A. Flick ${ }^{\text {a }}$, C. Gagliardi ${ }^{\text {, }}$, \\ E.V. Hungerford ${ }^{a, *}$, K. Johnston ${ }^{\text {a,e }}$, G.J. Kim ${ }^{d}$, K. Lan ${ }^{\text {a }}$, F. Liu ${ }^{\text {d }, ~ Y . C . ~ L u ~}{ }^{\text {a }}$, B.W. Mayes ${ }^{\text {a }}$, \\ R. Mischke ${ }^{c}$, R. Phelps ${ }^{\text {a,f }}$, L.S. Pinsky ${ }^{a}$, D. Semon ${ }^{\text {a,e }}$, R.E. Tribble ${ }^{d}$, L.G. Tang ${ }^{\text {a,g }}$, X.L. Tu ${ }^{d}$, \\ L. van Ausdeln ${ }^{\mathrm{d}}$, W. Von Witsch ${ }^{\mathrm{a}, \mathrm{j}}$, H.Z. Wang ${ }^{\mathrm{a}}, \mathrm{X}$. Yao ${ }^{\mathrm{a}, \mathrm{h}}, \mathrm{W}$. Yi ${ }^{\mathrm{k}}$ \\ ${ }^{\circ}$ Department of Physics, University of Houston, Houston, TX 77204, USA \\ ${ }^{b} 1814$ Belmont Lane, Redondo Beach, CA 90278, USA \\ ${ }^{c}$ MP-9 MS H846. Los Alamos National Lab, Los Alamos, NM 87545, USA \\ ${ }^{d}$ Cyclotron Institute, Texas A\&M Unversity, College Station, TX 77843, USA \\ ${ }^{e}$ Department of Physics, Louisiana Tech University, Ruston, LA 71272, USA \\ 'Department of Physics, University of Michigan, Ann Arbor, MI 48109, USA \\ ${ }^{8}$ Department of Physics, Hampton University, Hampton, VA 23668, USA \\ ${ }^{h}$ Tianjin Technical Physical Institute, Hongji Road-Jinnin Street, Dist. Nankal, Tianjin 300192, China \\ 'Department of Physics, Yale University, New Haven, CT 06511, USA \\ 'Department of Physics, University of Bonn, Bonn, Germany \\ ${ }^{k}$ Department of Physics, Virginia Polytechnical Institute, Blacksburg, VA, USA
}

Received 13 January 1994

The construction techniques used in the development of a set of large pair spectrometers for the MEGA detector are discussed. These spectrometers consist of carbon-fiber-composit cylinders on which the conversion foils are mounted, and appropriately spaced wire chambers in each layer which track the conversion leptons. Close tolerances on all dimensions are maintained. The detector has been operating well in the stopped muon beam line at LAMPF.

\section{Introduction}

One of the major challenges of particle and nuclear physics today is to understand the extent to which the minimal standard model (MSM) successfully describes the interactions among quarks, leptons and gluons, and to determine the modifications which are needed to construct a "complete" theory of the elementary particles and their interactions. The minimum standard model, MSM, SU(3) $\times \mathrm{SU}(2)_{\mathrm{w}} \times \mathrm{U}(1)$, of elementary particle interactions has been extraordinarily successful in describing a wide range of experimental observations. At present, there exists no confirmed result that violates it. The MSM, which includes six lepton flavors, six quark flavors with three colors each, a single Higgs doublet, and twelve gauge bosons, assigns the quark and lepton flavors to three "generations" of weak left-handed isodoublets and right-handed isosinglets.

+ Supported in part by the US DOE (Grant numbers DE-FG0587ER40310 and DE-FG03-93ER40765), NSF, and the Robert A. Welch Foundation.

* Corresponding author, tel +17137433550 , fax +1713743 3589.
In the MSM, all neutrinos are massless since they are purely left-handed particles. This leads to the conclusion that the neutrino weak eigenstates are also mass eigenstates, and there is no neutrino mixing. With these exceptions, the masses, mixing angles, and coupling constants of the MSM are arbitrary parameters, each of which must be determined independently from experimental observation. Within the MSM, baryon number and lepton number are all strictly conserved quantities.

In 1985, several groups formed a collaboration, MEGA, to undertake a new experimental search for the rare decay mode of the muon, $\mu \rightarrow \mathrm{e} \gamma$. As a lepton-flavor violating process, the branching ratio for this decay is sensitive to the underlying symmetries and/or substructures which manifest themselves in the observed generations. Previous attempts $[1,2]$ to observe this decay used $\mathrm{NaI}$ detectors to measure photon energies, together with either a magnetic spectrometer or $\mathrm{NaI}$ to measure positron energies. The present limit on the branching ratio is $<4.9 \times 10^{-11}$. In these measurements, the dominant background comes from random conicidences between high energy positrons from normal muon decay, $\mu \rightarrow \mathrm{e}^{+} v v$, and high energy photons from inner Bremsstrahlung muon decay, $\mu^{+} \rightarrow \mathrm{e}^{+} \gamma v v$. These backgrounds may be suppressed by high resolution 
coincidence measurements of the $\mathrm{e}^{+}$and $\gamma$ energies and directions. This is the approach applied by MEGA. Muons from the stopped muon channel, SMC, at the Los Alamos Meson Physics Facility, LAMPF, are stopped in a thin polyethylene target at the center of a $15 \mathrm{kG}$ magnetic field produced by a superconducting solenoid. Decay positrons are tracked through a set of eight cylindrical multiple-wire drift chambers, DC's, multiwire proportional chambers, (MWPC's). Decay photons are detected by a set of three independent, concentric, cylindrical pair spectrometers, which include drift chambers, MWPC's, and plastic scintillators, surrounding the positron detectors. The magnetic field serves both to measure the positron and photon momenta, and to confine the positrons from the normal decay $\mu \rightarrow$ evv to a small volume in the center of the solenoid. This keeps the photon detectors relatively quiet, in spite of the high instantaneous muon decay rate $(5 \times$ $\left.10^{8} \mathrm{~s}^{-1}\right)$ in the stopping target. The MEGA detector design provides much better energy and angular resolution for both the photon and positron than previous experiments and, coupled with its FASTBUS read-out system, the ability to operate at much higher rates. The ultimate goal of the MEGA experiment is to observe the decay $\mu \rightarrow \mathrm{e} \gamma$, if possible, or to set a $90 \%$ confidence limit on the branching ratio for this decay of a few parts in $10^{13}$, representing an improvement of a factor of about 200 compared to our present knowledge. Furthermore, MEGA is designed to be background-free at this level, whereas the previous experimental limit [2] required a maximum likelihood analysis, since there were background events observed in that measurement.

\section{Overview of the detector}

The pair spectrometers determine the momentum for photons and provide the trigger for the experiment. In order to have high solid angle acceptance, the size of the spectrometers requires a large volume of uniform magnetic field. The LASS magnet [3], a superconducting solenoid of $2 \mathrm{~m}$ bore and $2.9 \mathrm{~m}$ length, was moved to Los Alamos and installed at the SMC for the experiment. Extensive Monte Carlo studies were employed to develop a design for the pair spectrometers to maximize their efficiency, and their position and momentum resolutions. By using multiple spectrometers, one is able to maintain excellent momentum resolution while obtaining a good overall detection efficiency. Although the choice of pair spectrometers for photon detection implies that the acceptance is less that in previous efforts, the significant improvements obtained in energy, direction, and time resolution more than compensate. In addition, the high granularity of the MEGA detector, coupled with its FASTBUS readout system, permits operation at much higher muon stopping rates than previous experiments. The primary hardware trigger for the experiment is provided by a coincidence between patterns of hits in the MWPC and plastic scintillators. The patterns of this first stage trigger can be chosen to maximize the acceptance for photons with a particular transverse momentum. A second stage hardware trigger uses the hits in the drift chambers to check for patterns representing high energy photon conversion in the pair spectrometers. Details of the construction and operation of the two trigger systems will be discussed in a separate publication.

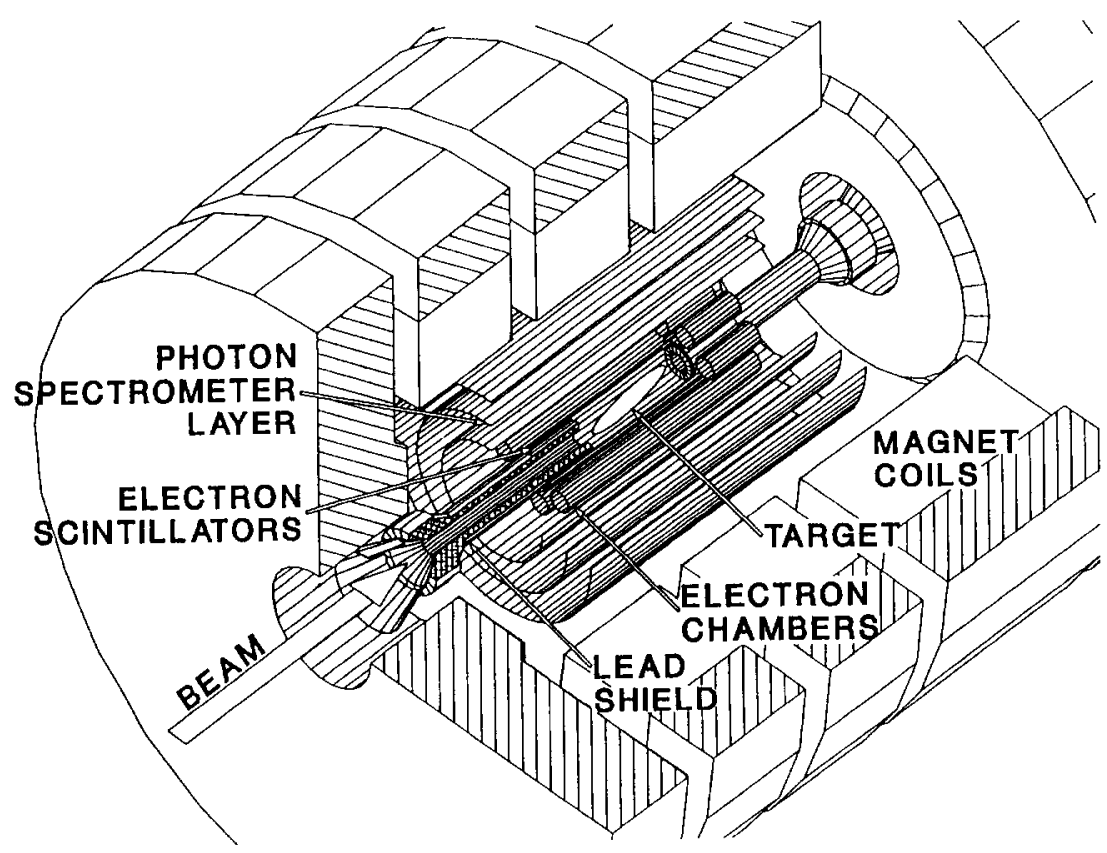

Fig. 1. A schematic layout of the MEGA Detector showing the magnet, electron and photon detectors, and the target. 
The general layout of the MEGA detector is shown in Fig. 1. There are three photon pair spectrometers (layers) which, except for size and a few details, are constructed in a similar manner. The positron spectrometer, which is not discussed here, lies inside the pair spectrometers. A surface muon beam is brought to rest in a thin, slanted target foil, and the particles from the decays are tracked by the detector. The major components of each pair spectrometer consist, from smaller to larger radius, of a scintillation barrel, a cylindrical conversion foil, a multiwire proportional counter (MWPC), another conversion foil, a delay line for $Z$ position measurement, a turning space with drift chambers (DC), and an outer aluminum cylinder for structural support and gas seal (CAN).

The scintillator bars provide timing and some axial position information, as well as help in pattern recognition of the event. Each conversion foil is composed of $0.25 \mathrm{~mm}$ $\mathrm{Pb}$ alloy supported by a carbon fiber composite cylinder, and covered with at least a $1.8 \mathrm{~mm}$ low mass foam layer. The foam thickness provides space for annihilation radiation pairs to circle without entering the active region of the wire chambes. A typical event showing one orbit of the conversion pair is shown in Fig. 2. The MWPC is used for pattern recognition, deconvoluting the multiplexed readout scheme, and identifying the conversion foil in which the pairs were produced. The delay line, positioned on the surface of the outer conversion cylinder, measures the axial position of a hit in the drift chamber just above it, by measuring the time difference of the induced charge as it appears at the ends of the line [4]. Then the meantime of the hits at the two ends of the lines determines the drift time in the cell. The turning space and DC's provide tracking to measure the perpendicular component of the momentum of the conversion pair. The outer aluminum CAN supports the tension of the chamber wires, provides a surface for the layup of the scintillators of the next layer, acts as an absorber to reduce the orbital radii of pairs so that they do not overlap the initial trajectories on subsequent passes of a pair through the spectrometers, and provides an outer gas seal for the detector.

The radii of the components in the three different spectrometer layers are given in Table 1 . The detector radii and the position of the first three drift chambers are selected to optimize the detector efficiency for $52.8 \mathrm{MeV}$ photons, which would be the photon energy for the $\mu \rightarrow$ e $\gamma$ decay. Note, however that layer 3 has four drift chambers and two additional grids of charge sweeping wires. This layer was designed specifically to be more sensitive to photons up to $70 \mathrm{MeV}$. Such photons, coming from $\pi^{\circ}$ decay, are used for calibrating the response of the detector to a known photon flux. While a lepton of the pair frequently has part of its orbit in the turning region of the spectrometer below the one in which the conversion occurred, it would not activate any sense wires of that detector. In addition as stated above, most pairs from annihilation radiation are excluded from the active region

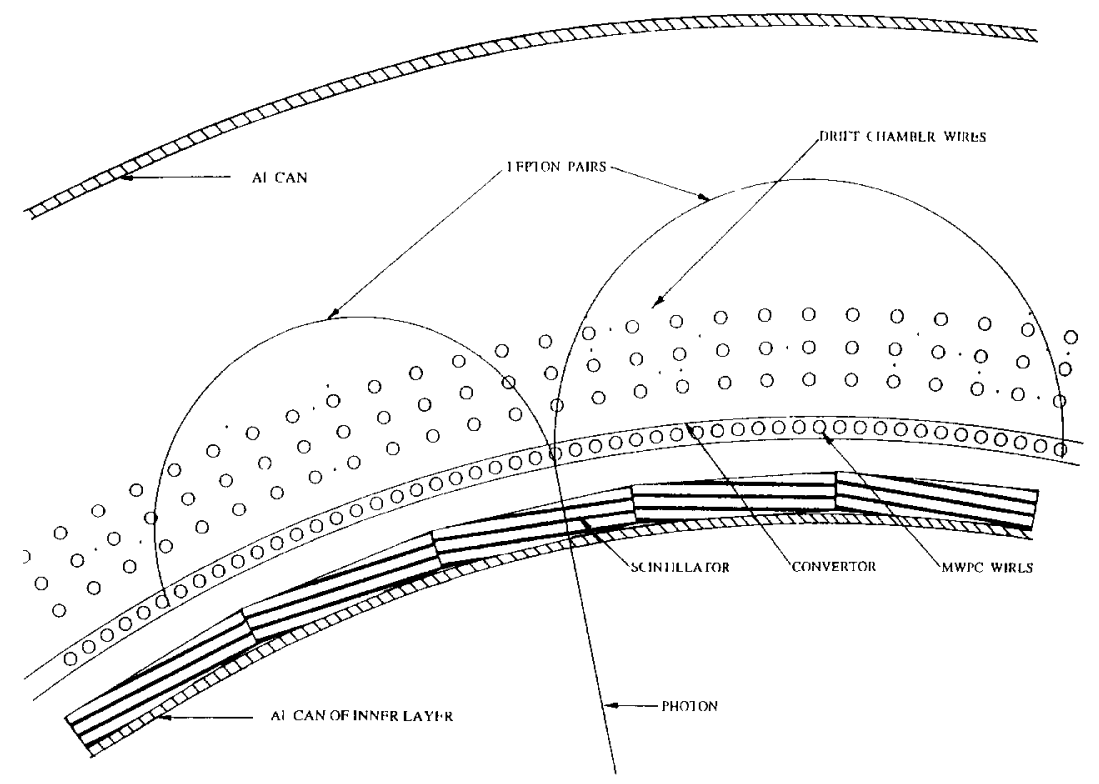

Fig. 2. A typical photon conversion showing the first half-orbit of the conversion pairs through the detector. The conversion, as shown, occured in the inner conversion cylinder. 
Table 1

Radii of different components in the pair spectrometer (detector length was $180 \mathrm{~cm}$ )

\begin{tabular}{lccc}
\hline & Layer 1 & Layer 2 & Layer 3 \\
\hline Scintillator & & & \\
$\quad$ Number & 40 & 60 & 80 \\
$\quad$ Radius [cm] & 32 & 47.9 & 63.4 \\
Conversion cylinder & & & \\
$\quad$ Radius of 1st [cm] & 33.6 & 49.5 & 64.9 \\
$\quad$ Radius of 2nd [cm] & 34.5 & 50.4 & 65.8 \\
MWPC & & & \\
$\quad$ Number of wires & 416 & 640 & 832 \\
$\quad$ Radius [cm] & 34.2 & 50.1 & 65.5 \\
Drift chamber 1 & & & \\
$\quad$ Number of wires & 208 & 320 & 416 \\
$\quad$ Radius [cm] & 35.3 & 51.3 & 66.7 \\
Drift chamber 2 & & & \\
$\quad$ Number of wires & 208 & 320 & 416 \\
$\quad$ Radius [cm] & 36.1 & 521 & 67.5 \\
Drift chamber 3 & & & \\
$\quad$ Number of wires & 208 & 320 & 416 \\
$\quad$ Radius [cm] & 36.9 & 52.9 & 68.3 \\
Drift chamber 4 & & & \\
$\quad$ Number of wires & - & - & 416 \\
$\quad$ Radius [cm] & - & - & 71.1 \\
\hline
\end{tabular}

of the wire chambers. The following sections describe the mechanical design and construction of the pair spectrometers in detail.

\section{Mandrel construction}

The conversion foils were mounted on cylinders constructed on a mandrel which was manufactured on a large lathe-type machine built specifically for this purpose. Fig. 3 shows the lathe, mandrel, and a typical cylinder, mounted on the machine. As six cylinders of different dimensions, two for each layer, were needed, the mandrel was required to be inexpensive and easily adapted to different radii, while allowing for precision tolerances on the diameter,

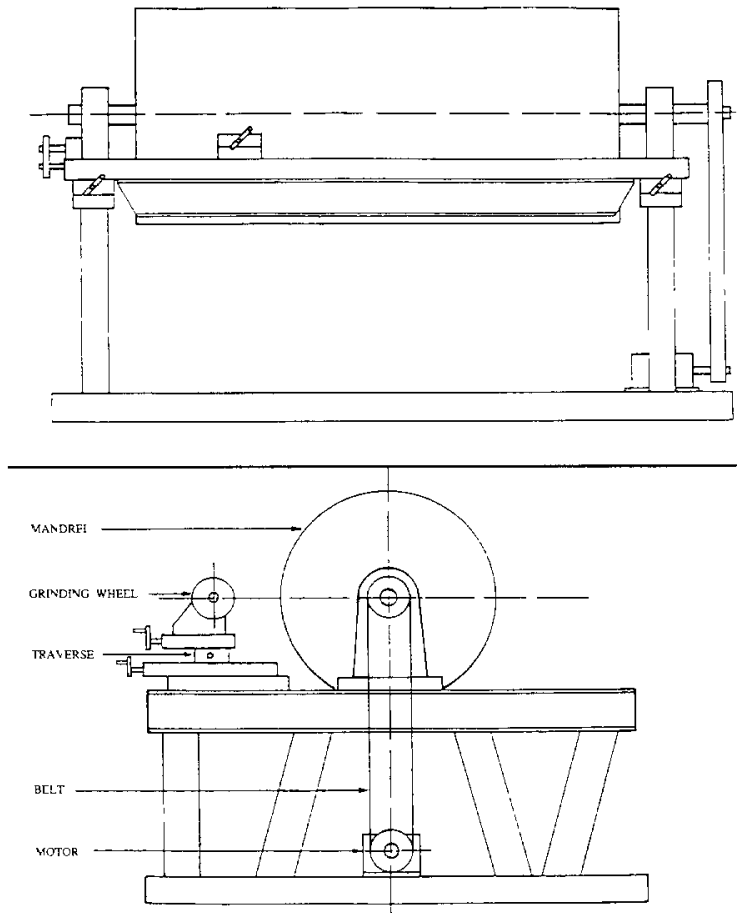

Fig. 3. The Lathe-type machine used to construct the conversion cylinders. A mandrel for cylinder layup is shown, mounted on the machıne.

and smoothness of the surface. Thus the mandrel was constructed of foam, fiberglass, and $1.6 \mathrm{~mm} \mathrm{Al}$ ribs mounted on a $12.5 \mathrm{~cm}$ steel pipe of $1.5 \mathrm{~cm}$ wall thickness.

A cross section of the mandrel is shown in Fig. 4. The outer surface of the mandrel was composed of a sandwich of fiberglass and foam about $5 \mathrm{~mm}$ in thickness. A channel of $8 \mathrm{~mm}$ width ran the length of the mandrel in the foam between these fiberglass layers into which a saw blade was inserted later to cut the completed cylinder off the mandrel. The outer fiberglass layer formed a hard surface on which the cylinder was constructed. This surface was waxed so that any epoxy which leaked onto it from the

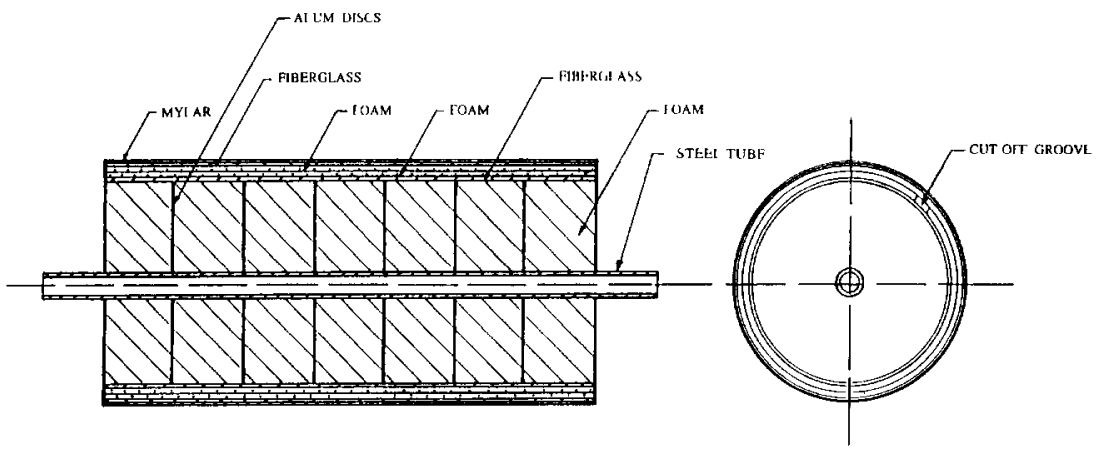

Fig. 4. The cross section of the mandrel used to construct the conversion cylinders. Note the surface is composed of a fiberglass-foam sandwich which allows the outer fiberglass layer with the cylinder to be cut off the mandrel after completion. 
construction of the cylinder would not bond to it. The surface was then spiral wrapped by a layer of $0.1 \mathrm{~mm}$ mylar. It was to this mylar layer that the inner foil was temporarily attached as described in the section on foil preparation. The surface of the mandrel was machined to the correct diameter by a diamond grinding wheel [5] mounted on a tool post grinder. The grinder moved along a motor driven traverse [6] while the mandrel was rotated by a variable speed, reversible motor. Positioning accuracy of the traverse was checked to be $25 \mu \mathrm{m}$ over the length of movement. As a "rule of thumb" the grinder was set to remove 0.3 linear meters of material per second. The system remained vibration free and was capable of reproducing a surface to about $100 \mu \mathrm{m}$ in the diameter. Alignment of the traverse was accomplished by measuring the diameter of the machined mandrel at several positions along its axis. The ends of the traverse were then adjusted so that it was parallel to the axis of the cylinder. Radial movement was accomplished by micrometer adjustment of the grinder frame. Reproducibility was found to be at the $25 \mu \mathrm{m}$ level.

\section{Cylinder construction}

A cross section of a conversion cylinder is shown in Fig. 5. The cylinders support the lead conversion foils and the delay lines, and act as electrostatic surfaces and a gas barrier between the MWPC and the drift chambers. A gas barrier was needed since the two detector types are operated with different gas mixtures. These requirments mandated a sturdy, low-mass cylinder which would minimally disturb the orbits of the conversion pairs, but have circular cross section to high precision. The fact that the cylinders were not required to support wire tension was critical in keeping them circular. This point is emphasized in Appendix A, where calculations of cylinder deformations, with the constraint that the ends of the cylinder retain

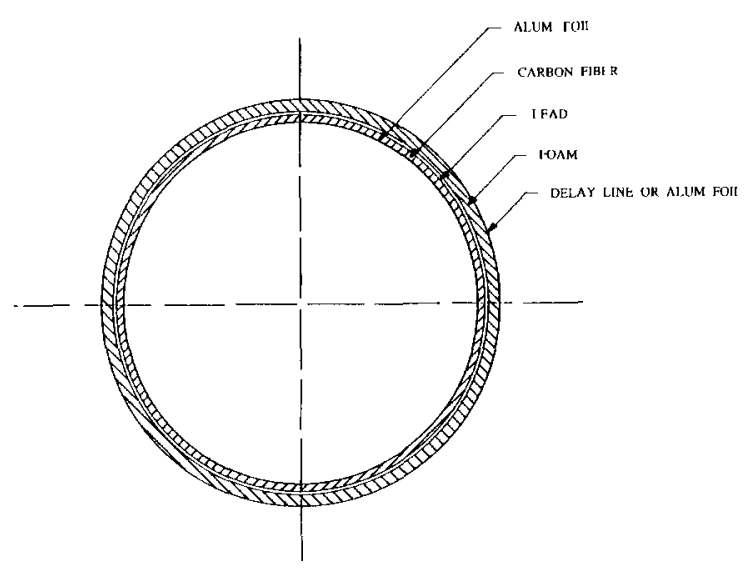

Fig. 5. A cross section of a conversion cylinder.

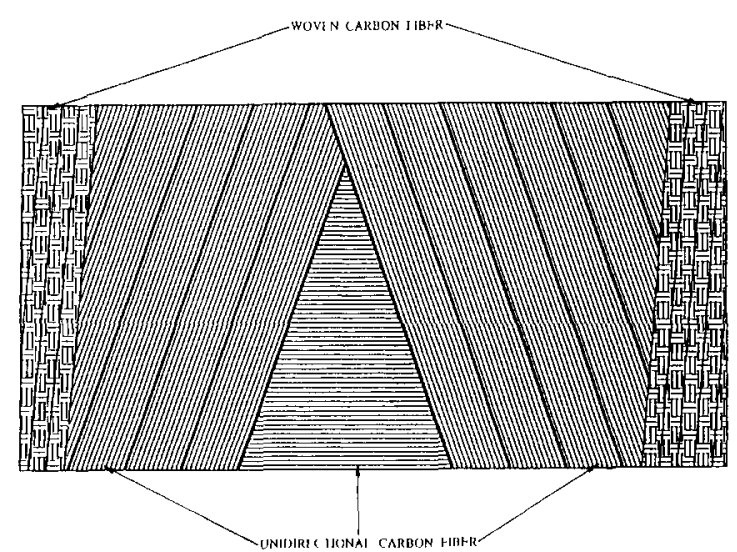

Fig. 6. Unuaxial Carbon fiber cloth in $12 \mathrm{ft}$ wide tape was wound on the mandrel as shown to form the support structure for the cylinder. The outer layer of woven carbon fiber tape was applied

to compress and hold the layers together while the epoxy set.

circular shape, are shown. These calculations indicate that deviations from circular cross section are negligibly small, including sag due to gravity, for cylinders without seams or joints, using materials of modest moduli. The MEGA cylinders are manufactured from carbon fiber cloth, with the fiber oriented in different directions and held in place by epoxy resin. The resulting composite material has low mass, low atomic number, and great stiffness. In this way the dependence of the cylinder on defects is greatly minimized. The design goal is to hold deviations from roundness of the finished product to about $75 \mu \mathrm{m}$ in radius.

Fig. 6 shows how the various carbon fiber layers were applied. The carbon fiber was uniaxial filament cloth [7], $0.18 \mathrm{~mm}$ thick. The spiral wraps provide hoop stiffness, and the axial layup provide stiffness in the axial bending moment. The spiral wrap could have been applied by winding carbon filiment directly onto a mandrel. In fact this was cone for the smaller cylinder of layer 2 , which was the first cylinder constructed. However it proved easier and just as effective to use carbon uniaxial cloth, which could be applied much more quickly by hand. All other cylinders were constructed from the uniaxial cloth.

The bonding epoxy was Hexcell 2410 with hardener 2183 [8]. An exhaustive study of epoxies was not made, but the product used here had good viscosity, working time, and strength. Several other types were investigated but were found to be too viscous, and in some cases set too quickly. After the uniaxial fiber layup, a layer of $5 \mathrm{~cm}$ woven carbon fiber tape [9] was wound by hand onto the cylinder. This tape was applied with tension to compress the underlying layers and hold them under compression as the epoxy set. It was found that a tape of woven carbon cloth was the best choice for this purpose, since it was porous and allowed excess epoxy to escape through the cloth as the tension was applied. Although some of this tape was ground away to create a smoooth surface for the 
lead layup, the remainder added strength to the cylinder. The cured cylinder formed a seamless carbon composite, approximately $1 \mathrm{~mm}$ thick, with a density of about $1.2 \mathrm{~g} / \mathrm{cm}^{3}$.

The lead was placed on the cylinder in axial sheets. It was composed of an alloy [10], which provided some stiffness as pure lead was found to be too soft, deforming under its own weight. Since the lead was rolled to the correct thickness by the manufacturer, the edges of the sheets did not lie completely flat. Thus about $3 \mathrm{~cm}$ was removed on each side of the sheets. These cuts created a straight edge on the sheet which allowed them to be placed together, edge on edge, without a visible seam.

The lead sheets were converted into panels for layup as described in the section on foil preparation. In summary two lead sheets, each $40 \mathrm{~cm}$ wide and about $175 \mathrm{~cm}$ long were placed together on a large sheet of mylar, $0.18 \mathrm{~mm}$ thick, which had been sprayed with an artist's adhesive [11]. The structure was then placed in a vacuum bag so that the lead was flattened and attached to the mylar by the adhesive. After a few minutes this structure was removed from the vacuum bag and applied to the cylinder.

Before application of the lead to the carbon composite, the cylinder was ground smooth with a diamond grinding wheel, cooled by water spray. The water spray also helped to control the composite dust released in the grinding, which can be toxic. A special epoxy [12] was used which had acceptable adhesive properties for a composite to metal bond. The lead panels were applied to the cylinder and held under compressional tension until the epoxy set by winding $2.54 \mathrm{~cm}$ mylar tape under tension around the cylinder. After the epoxy bond formed, the process was repeated with additional panels until the cylinder was covered by the lead foil.

A layer of polyurethane foam [13] was then applied by placing $180 \mathrm{~cm} \times 61 \mathrm{~cm} \times 0.6 \mathrm{~cm}$ foam sheets over an epoxy layer applied to the lead surface. The foam was held under compressional tension until the epoxy set by mylar tape as described above. After the epoxy had cured the foam layer was ground to the correct dimensions for the cylinder radius, less the final covering, which was either an aluminum foil or a delay line depending on the type of cylinder. The aluminum foil and the delay lines were applied in the same manner as the lead foil. Care was taken to completely cover the foam surface with epoxy since polyurethane is known to accelerate aging of wire chambers [14]. Also note that the foam surface was essentially covered by either an aluminum foil or a delay line which protected the surface from the chamber gas. To check the susceptibility of this foam to chamber gas, pieces of bare foam were placed in the operating gas mixture for 2 months. No dimensional changes of the pieces were found.

The completed cylinder was allowed to cure for a few days, cut to length, and then removed from the mandrel. This was accomplished by running a band saw blade

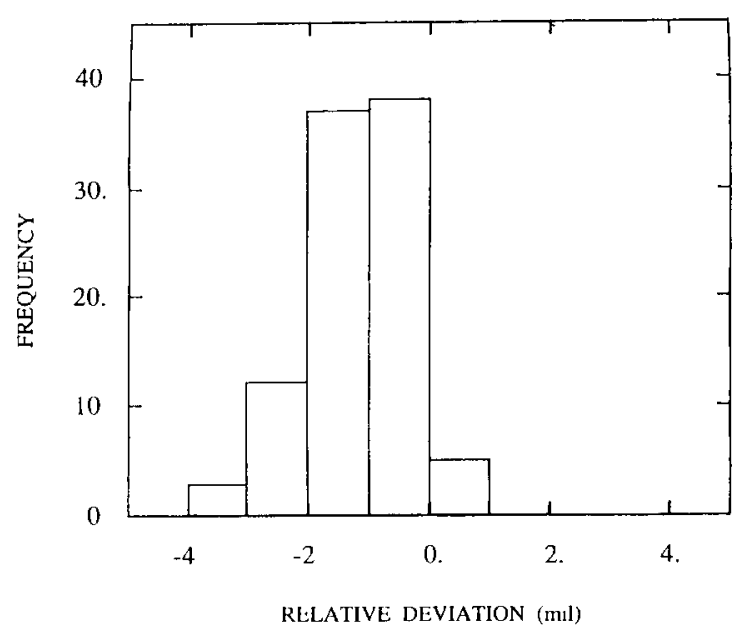

Fig. 7. The frequency of measured deviations from round of a cylinder sampled at regular intervals about the length and circumference

through a channel in the mandrill. The blade was attached to an apparatus resembling a large hack saw and a circumferential cut was made through the foam of the mandrill, between the two fiber glass layers near the surface. Thus a gap of approximately the width of the saw blade was created around the mandrel. Removal of the cylinder from the mandrel would have been easiest if sufficient height in the laboratory had been present to extract the cylinder vertically. As this was not the case, the mandrel was supported horizontally by a long, stiff beam. Mylar sheets were inserted in the gap created by the saw cut and the cylinder pushed, sliding on this mylar layer, onto a wooden spline of appropriate dimensions. The cylinder was then turned vertically using the spline for support, and the remains of the mandrel outer wall removed from the interior. Before final assembly the surfaces of the cylinder were cleaned of adhesives including epoxy which had leaked through the seams.

Fig. 7 shows the deviation of a typical cylinder from a circular cross section. These measurements were made by sampling positions on the cylindrical surface using an indicator gauge mounted on a slide which was positioned on a precision granite table. The cylinder was measured with the axis horizontal and the ends of the cylinder inserted into the end caps. Reproducibility was found to be at the level of $25 \mu \mathrm{m}$ which is also the level of uncertainty in the measurements.

\section{Foil preparation}

A vacuum bag technique was used to produce panels of aluminum foil, lead foil, and delay lines for hand layup on the cylinder. This technique used a $122 \mathrm{~cm} \times 244 \mathrm{~cm}$ precision granite block as a layup surface. A panel consisting 
of foils preliminary tacked to a backing of $0.25 \mathrm{~mm}$ mylar by artist's adhesive was placed on the granite block and this panel covered by another sheet of $0.25 \mathrm{~mm}$ mylar. Typically two foils, each about $25 \mathrm{~cm}$ wide were aligned edge to edge on one backing, although as many as three foils were attached in some cases. The limit to the number of foils on a backing was mainly one of ease in handling the finished panel. A $3 \mathrm{~mm}$ nylon cord was taped under the cover sheet near its outer edge, to ease the escape of trapped air as it was pumped away. The cover sheet was taped to the surface of the granite block using celophane tape. A small vacuum pump then removed the air between the block and the cover sheet by pumping through a small hole in this sheet. After a few minutes the foils were flattened and firmly attached to the mylar backing. The panels were then removed from the bag and transferred to the cylinders. After the foils were attached to the cylinder by epoxy, the backing was easily peeled away.

The Aluminum foil was $50 \mu \mathrm{m}$ thick and was manufactured from 1145 aluminum alloy [15] of hardness 19. It was found that this material had the appropriate hardness to form a smooth surface, and was relatively easily handled without the introduction of wrinkles. The material was rolled to the final thickness and sheared to the correct width by the manufacturer. However, as with the $\mathrm{Pb}$ foil, it was found that the foil did not lie flat due to stretching of the edges during the rolling and shearing process. Thus about $3 \mathrm{~cm}$ of the width on each side was trimmed away. The inner aluminum foil of each cylinder, which was mounted directly on the mandrel was attached to mylar wound on the mandrel surface. Except for the use of the
Table 2

Delay line characteristics

\begin{tabular}{lcc}
\hline & Layer 1 & Layer 2-3 \\
\hline Cu trace thickness & $0.25 \mathrm{~mm}$ & $0.25 \mathrm{~mm}$ \\
Acrylic adhesive thickness & $0.25 \mathrm{~mm}$ & $0.25 \mathrm{~mm}$ \\
Kapton substrate & $0.76 \mathrm{~mm}$ & $0.76 \mathrm{~mm}$ \\
Trace to trace spacing & $0.25 \mathrm{~mm}$ & $0.25 \mathrm{~mm}$ \\
Trace width & $979 \mathrm{~mm}$ & $9.24 \mathrm{~mm}$ \\
Strip spacing & $0.76 \mathrm{~mm}$ & $076 \mathrm{~mm}$ \\
Total length & $173.8 \mathrm{~cm}$ & $173.8 \mathrm{~cm}$ \\
Total trace length & $3404 \mathrm{~cm}$ & $3218 \mathrm{~cm}$ \\
DC resistance & $72 \Omega$ & $72 \Omega$ \\
Characteristic impedance & $110 \Omega$ & $110 \Omega$ \\
One way transit time & $253 \mathrm{~ns}$ & $244 \mathrm{~ns}$ \\
$\quad$ total length) & $13.5 \mathrm{~cm} / \mathrm{ns}$ & $132 \mathrm{~cm} / \mathrm{ns}$ \\
Propagation speed & & \\
$\quad$ (along trace) & $50 \%$ & $50 \%$ \\
Total attenuation &
\end{tabular}

vacuum hold down, the procedure for mounting these inner aluminum foils was the same as described above.

\section{Delay lines}

The delay lines $[4,16]$ were etched on both surfaces of a polyamide laminate in groups of 27 . Although it was possible to manufacture delay lines of the required $174 \mathrm{~cm}$ length, it was difficult to obtain polyimide laminates which resulted in lines with low dispersion. Thus delay lines of
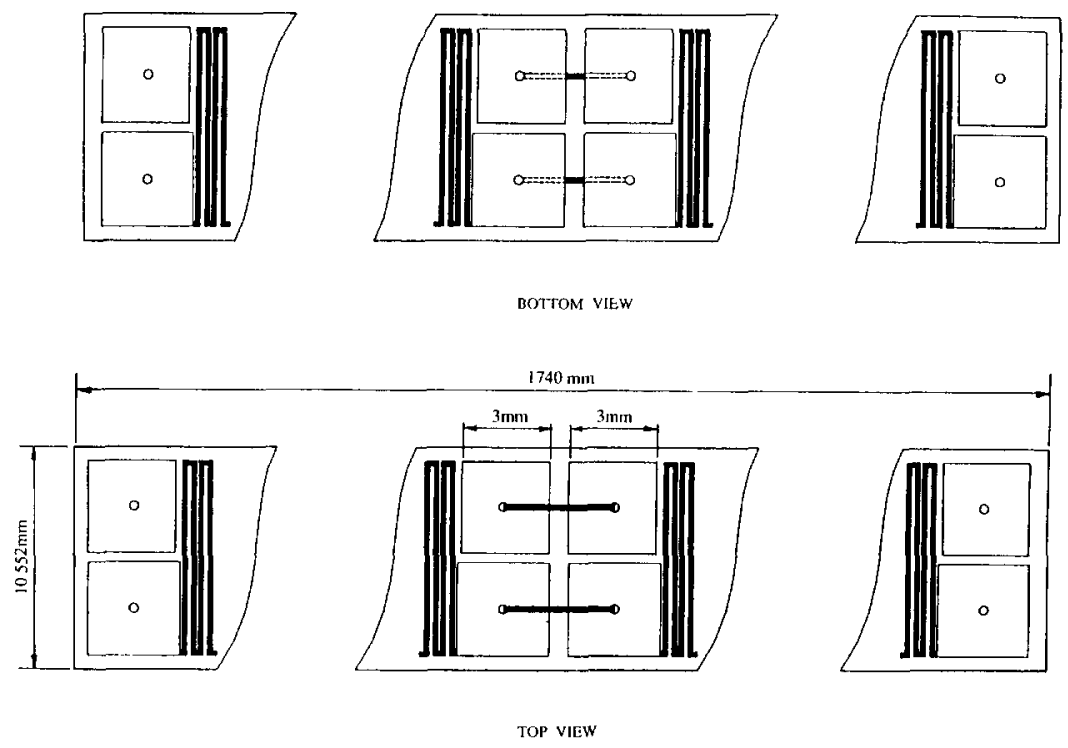

Fig. 8. A schematic of a delay line strip showing that the top and bottom trace are out of phase by $180^{\circ}$, and how the line was jorned at the centers. 
half the required length were manufactured and these panels spliced together to form a $174 \mathrm{~cm}$ length. A schematic geometry of a delay line is shown in Fig. 8. Each line terminated in a small pad which allowed interconnections between pannels and top to bottom connections. As indicated, the zig-zag etch pattern was shifted between top and bottom surfaces to increase the inductance. Table 2 gives the characteristics of the delay lines. It was found that about $10 \%$ of the lines had a manufacturing flaw represented by a break, a delamination, or an unetched section. Most of these flaws were repaired with either solder or conducting epoxy [17].

After continuity checks the pannels were trimmed and joined to form the $174 \mathrm{~cm}$ length needed to cover the cylinder. It was found that the electrical connections between panels could be made by soldering 28 gauge wire wrap wire rolled to $250 \mu \mathrm{m}$ thickness and inserted through holes drilled into the flats on the delay lines. The wire ends were pushed into the foam layer as shown in the figure. The panels were carefully aligned so that the axis of the delay lines in each segment were parallel. Tolerances of $124 \mu \mathrm{m}$ on this alignemnt were held.

The gap between individual delay lines was $0.75 \mathrm{~mm}$. This width of material at the edges of each panel was removed, to allow some placement adjustment, so that position under the drift chamber sense wire could be maintained. In order to align the delay lines axially on a cylinder, $1 \mathrm{~cm}$ wide strips of surplus delay line were joined and adjusted so that the total number of lines fit circumferentially around the cylinder. These were temporarily attached to the ends of the cylinder and aligned with each other using the traverse on the layup lathe. Then panels of delay lines were placed on the cylinder, visually aligned with these delay line strips, and attached with epoxy. The precision of the placement of the lines was $\pm 0.25 \mathrm{~mm}$.

\section{Construction of the end caps}

The end caps are constructed from tooling plate Aluminum alloy, $3.4 \mathrm{~cm}$ thick. They are machined from one plate in the case of layers 1 and 2 . For layer 3 they are composed of four segments since the diameter of the end caps for this layer was larger than the standard width of $\mathrm{Al}$ plate. These four segments were bolted and epoxied together. All machining and drilling was handled by a numerically controlled mill. Tolerances were held to the $25 \mu \mathrm{m}$ level, which required monitoring the temperature of the plates during the maching process. Feedthrough holes for the wires were drilled and reamed.

Grooves for the cylinders were machined so that the inner edge of each groove provided the aligning surface by mating with the hard, graphite, inner surface of each cylinder. The outer groove was cut $8 \mathrm{~mm}$ deeper than the inner groove so that upon assembly the outer cylinder, which was made longer, was inserted first. Assembly is discussed in section 8 .

Layer 1 was constructed so that the inner cylinder was mated to precision rings. This assembly then slid into the end caps with the rings providing the positioning dimensions. The gas seal was provided by a $0.75 \mathrm{~mm}$ ' $O$ ' ring. This design greatly facilitated assembly and provided the possibility that the system could be partially disassembled at a later date. However it was much more expensive to fabricate, and for that reason it was not implemented for layers 2 and 3 .

The end caps were held apart by an outer Al cylinder, CAN, of $2 \mathrm{~mm}$ thickness, which was rolled and welded into a cylindrical shape. Precision in circular shape was not required for this CAN. It was placed in rings which slipped over the edge of the end caps. The gas seal was obtained by an ' $\mathrm{O}$ ' ring in the end caps. The CAN completely supported the wire tension. It was covered with a layer of foam and was ground to the correct cylindrical dimensions to support the scintillators used for the next pair spectrometer layer. The layup of the scintillators is described in section 7 .

Gas was supplied to the MWPC through a gas manifold, distributing the gas through a system of $1 \mathrm{~mm}$ stainless steel and teflon tubes about every $8 \mathrm{~cm}$ in circumference about the detector. The gas manifold was a $9 \mathrm{~mm} \mathrm{Cu}$ pipe attached to the end cap. Gas to the drift chambers and turning regions was supplied through four gas holes in the end cap.

\section{Assembly}

The detector was assembled by first epoxying the larger of the two conversion cylinders into one end cap. This cylinder had delay lines on its outer surface and therefore after placement in its groove, it was aligned with respect to the sense wire holes in the end cap. Care was taken to assure this cylinder was aligned vertically as the epoxy set. This was accomplished by temporarly placing the upper end cap on the cylinder and aligning the edges of the end caps. Conducting epoxy [17] was used to electrically connect the MWPC foils and the inner lead conversion foil to the end cap. Just enough epoxy was used for a gas seal and a strength bond.

After the outer cylinder was fastened to an end cap, the inner cylinder was lowered into the outer cylinder using a crane and supporting fixture. This cylinder was epoxied in place as described previously. Vertical alignment in this case was accomplished by spacing the inner cylinder $6 \mathrm{~mm}$ inside the outer cylinder at various positions around the circumference.

Then the other end cap was inserted and the detector inverted. The end cap with the cylinders attached was raised, epoxy placed in the end cap grooves, and the two components mated. The outer cylinder being longer than 


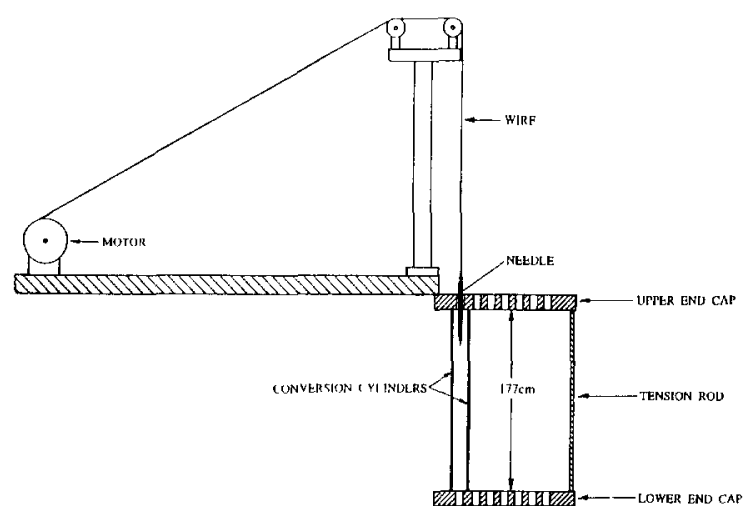

Fig. 9. A schematic drawing of the wiring apparatus. The plate on which the motor and pulleys was mounted pivoted about the center of the chamber and was indexed to the wire holes for alignment.

the inner one, allowed each cylinder to be inserted separately into the end cap as the upper component was lowered. Again care was taken to align the delay lines with the appropriate sense wire holes in the end cap.

In layers one and two, four tension rods were inserted between the end caps to provide the correct spacing between them, and to support the wire tension as the detector was wired. These were removed after the outer CAN was in place. Finally the detector was wrapped with mylar for protection and was mounted for wiring. After wiring, the detector was removed from its stand and the outer CAN slipped into place. A ring attached to each end cap was placed over the lip of the CAN and tension screws, counter sunk into the ring, used to transfer the wire tension from the tension rods to the CAN. Indicator gauges positioned at four places on the end cap were used to show when the tension was transferred to the screws. When the CAN supported the wire tension the tension rods were removed and the holes plugged.

In the case of layer 3 , the end caps were separated by eight equally spaced, Aluminum, tension rods, $1.9 \mathrm{~cm}$ in diameter. These rods were positioned at the outer edge of the end caps and were left in place to support the wire tension. This layer was treated differently due to the tension from the larger number of wires, the fact that the end cap was not solid but composed of four segments, and the fact that there was no spectrometer at larger radius.

Scintillators were positioned on the foam layer around the CAN and taped into place. Each layer was lowered into the other and connected to positioning spiders every $45^{\circ}$. The fully assembled system was inserted via rails into the LASS magnet and supported by legs on the vacuum can of the magnet coil. Care was taken to electrically isolate the detector from the magnet. The weight of the entire detector was approximately $850 \mathrm{~kg}$.

\section{Wiring}

After joining the cylinders to the end caps, the MWPC volume was enclosed between the conversion cylinders. However there was access to the drift chamber area as the CAN was not put in place until wiring was completed. Recall that the end caps are held apart by the tension rods. These rods are removed after the CAN is loaded with the wire tension. During wiring, the detector was enclosed by a cylinder of clear mylar to protect the interior DC wires as they were put in place, and to act as a dust shield.

The detector was mounted in a clean room on a tripod stand which held the lower end cap approximately $1 \mathrm{~m}$ from the floor. It was then leveled so that the wire holes in the top and bottom end caps were vertically aligned. The

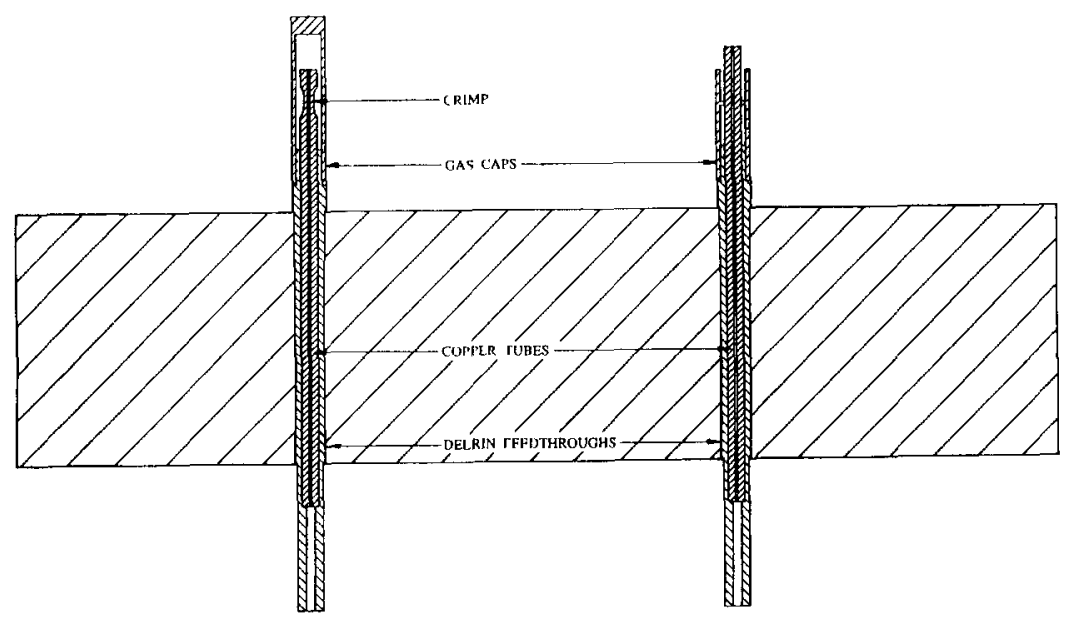

Fig. 10. The Delrin feedthroughs showing the Cu-tube crimp arrangement to pin the wires. Note the two types of gas caps, one allowing electrical connection to the wire. 
vertical clearance below the lower end cap allowed access to the bottom of this end cap. Access above was obtained by a platform constructed around the tripod stand.

The wiring apparatus consisted of a set of pulleys, a motor to unwind the wire from the spool, and a wiring needle. The needle was constructed of soft steel and weighed $5 \mathrm{gm}$ for the field wires and $3 \mathrm{gm}$ for the sense wires. As the motor turned the spool, the needle pulled the wire vertically through the holes in the end caps by gravity, Fig. 9. If properly aligned and if the detector was free from vibration, the needle passed through both holes without difficulty. Occasionally the wire did not unwind smoothly from the spool which produced small oscillations in the suspended needle. These in turn could cause the needle to miss the hole in the lower end cap. If this occurred the motor was reversed until the needle was again suspended just above the bottom hole. After a minute or so the oscillations were damped sufficiently for the needle to be lowered through the hole. There were rare instances when the wire broke dropping the needle inside the detector. If this happened in the drift chamber region it was easily retrieved. Although the MWPC formed an enclosed area, the needle could be retrieved in this case without too much effort using a small but strong magnet.

Wires [18] were held in the end caps by a feedthrough with an inserted copper tube, crimped about the wire. This system was designed for the crystal box experiment [2] and is shown in Fig. 10. It should position the wire within $\pm 40 \mu \mathrm{m}$. The copper tubes for the sense wires had an inner diameter of $100 \mu \mathrm{m}$ while the tubes for the field wires had inner diameter of $225 \mu \mathrm{m}$. The feedthroughs were injection molded using Delrin [19] into an existing die. Delrin was chosen for both its mechanical and electrical properties. After the wire had been threaded through the holes in the end caps it was inserted through a feedthrough and a copper tube. The upper tube was crimped to hold the wire in place. Then the wire was tensioned by hanging a weight on the wire and the lower tube crimped. It was found that on average about one sense wire could be run every 3 to $4 \mathrm{~min}$, and a field wire about 1 to $2 \mathrm{~min}$. Small surgical scissors were used to cut the wire without kinks or burrs.

The applied wire tension is given in Table 3. It was

Table 3

Wire tension (all wires $175 \mathrm{~cm}$ long)

\begin{tabular}{lcll}
\hline Wire & $\begin{array}{l}\text { Diameter } \\
{[\mu \mathrm{m}]}\end{array}$ & Material & $\begin{array}{l}\text { Tension } \\
{[\mathrm{g}]}\end{array}$ \\
\hline MWPC sense & 25 & W (Au coat) & 110 \\
DC1 sense & 25 & W (Au coat) & 110 \\
DC2 sense & 33 & W (Au coat) & 110 \\
DC3 sense & 33 & W (Au coat) & 110 \\
DC4 sense & 33 & W (Au coat) & 110 \\
DC field & 102 & CuBe & 250 \\
\hline
\end{tabular}

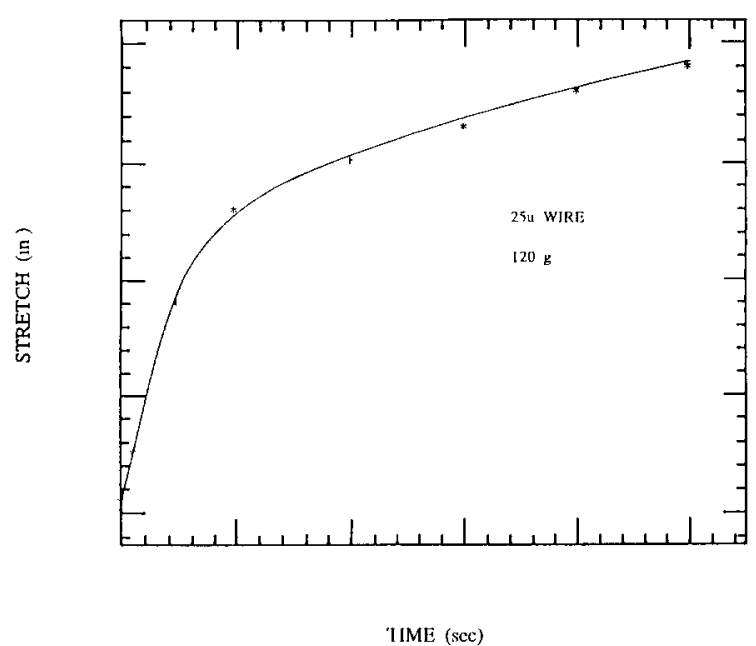

Fig. 11. Measured creep for a $25 \mu \mathrm{m}$ wire under a constant $120 \mathrm{~g}$ force.

chosen to be greater than the expected electrostatic forces [21]. The tungsten sense wire was found to experience creep over an extended period of time as shown in Fig. 11. The actual tension in the wire was found to be about $10 \mathrm{gm}$ less than the applied weight. Part of this reduction was due to this creep and part due to the crimping process. At this tension the wire obeyed Hooke's law as shown in Fig. 12.

The feedthrough-copper tube system was designed to be used with springs. For several reasons, and in particular since appropriate springs could not be easily found for this tension, they were omitted. The maximum gravitational sag for the $25 \mu \mathrm{m}$ and $35 \mu \mathrm{m} \mathrm{W}$ wire at $110 \mathrm{gm}$ tension was $33 \mu \mathrm{m}$ and $56 \mu \mathrm{m}$ respectively [25]. At $250 \mathrm{gm}$ the

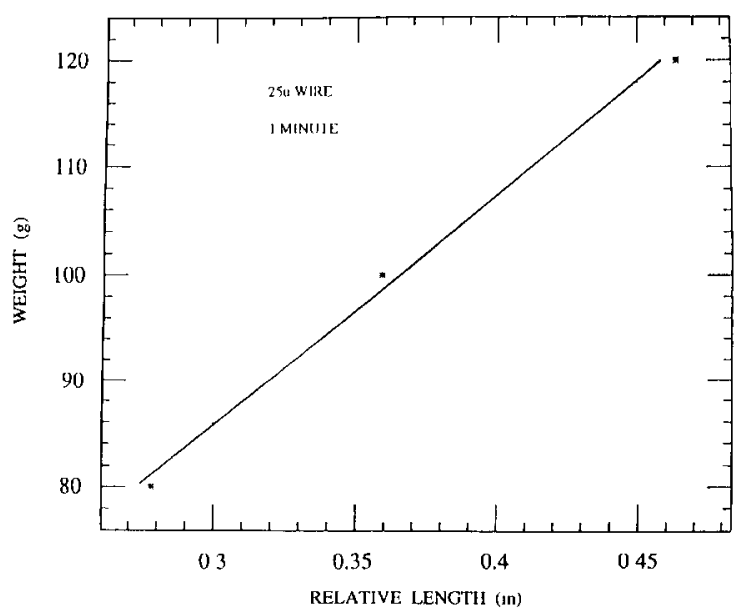

Fig. 12. The relative length of a $25 \mu \mathrm{m}$ wire as a function of applied force after $1 \mathrm{~min}$. The wire obeys Hooke's Law for this range of applied force. 
maximum sag of the $\mathrm{Cu}-\mathrm{Be}$ field wire was $120 \mu \mathrm{m}$, which was barely within tolerance, although this deflection was not expected to prejudice the operation of the wire chamber. However an increase of this wire tension would have been unacceptable due to the imposed stress between the end caps.

After wiring, less than $1 \%$ failure rate of the wires was experienced. These almost all occurred in the first week, and these wires were replaced before the system was removed from the stand. Gas caps were then placed over the feedthroughs. One side of the detector had caps with a tight fitting seal about the copper tubes, which protruded about $4 \mathrm{~mm}$ through the caps. The other gas caps enclosed the copper tubes in the gas seal. It was found that this system adequately provided a gas barrier in most instances. In some cases a small dot of Glyptal enamel [22], diluted with acetone, was required to complete the seal.

The voltage distribution grid was connected to the field wires by soldering 28 gauge, bare, solid conductor to the ends of the copper tubes on field wires. Readout contacts were made through female socket pins plugged onto the copper tubes on sense wires.

\section{Wire chambers}

The MWPC was somewhat unconventional in that the wire spacing is larger than the half gap. Greater definition of the pair position is not required, and more importantly, the width of the feedthrough would not allow closer spacing. The large wire spacing also allowed the chamber to operate without wire garlands which would have been difficult to implement. The sense wire diameter for the MWPC was $25 \mu \mathrm{m}$ and the cylinder walls provided

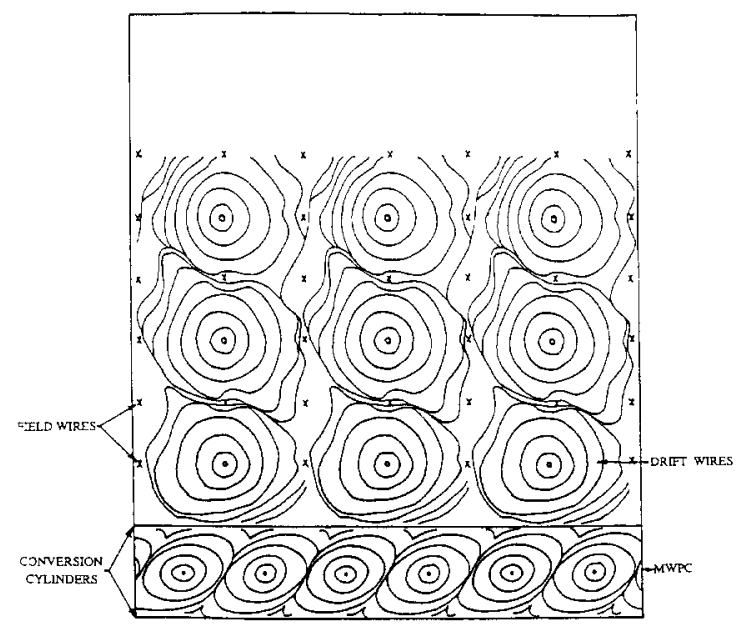

Fig. 13. Equi-drift time contours for the MEGA wire chambers in a $1.5 \mathrm{~T}$ magnetic field. Each contour represents $20 \mathrm{~ns}$ of drift time in an argon-ethane gas mixture. The gas mixture in the MWPC actually had $40 \%$ faster drift velocity

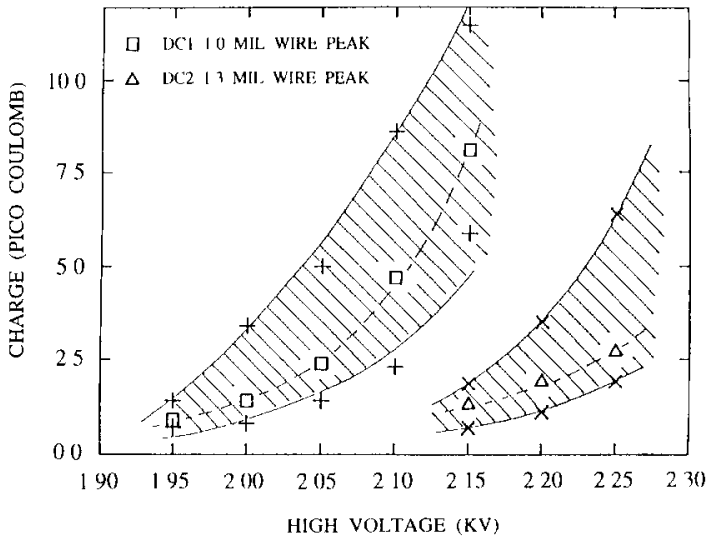

Fig. 14. A measured comparison of the collected charge for different wire diameters as a function of high voltage on the detector for the MEGA detector geometry

grounded electrostatic surfaces. Operational voltage was about $2100 \mathrm{~V}$. Wire tension as previously indicated was about $115 \mathrm{gm}$. Equal drift time contours are shown in Fig. 13 for the field on $(1.5 \mathrm{~T})$ configuration.

There are three drift chambers in layers 1 and 2 and four in layer 3 . They are placed near the surface of the outer conversion cylinder so that leptons from the conversion pair would traverse as many drift chambers as possible. In fact, one of the leptons from the pair must traverse all three drift chambers and the other must traverse at least two chambers in order for the conversion vertex to be determined reliably. Drift chamber 1 is read out only through the induced charge on the delay lines which lie on the surface of the outer conversion cylinder. The time difference between the appearance of the induced charge at the ends of the delay line is proportional to the position along the wire, while the time sum determines the drift time of the charge to the wire. The other drift chambers are conventional proportional chambers with approximately rectangular cell geometry shaped by field wires. The sense wire diameter was $25 \mu \mathrm{m}$ for drift chamber 1 and $33 \mu \mathrm{m}$ for the other drift chambers. The difference in wire diameter provides the relatively higher gain needed to read out the delay lines as opposed to the sense wires. Fig. 14 shows the relative gain between wires of various diameters for the argon/ethane gas mixture used. The field wires were $100 \mu \mathrm{m}$ in diameter. The field shaping wire grid is shown in Fig. 15. Operation of the drift cell was insensitive to the voltage on the corner wire, and it was kept at about $-250 \mathrm{~V}$. Table 4 gives the capacitance of a wire grid calculated as described in Appendix B. Operational voltage for the drift chambers was $2200 \mathrm{~V}$. Drift time paths are shown in Fig. 16 for field "on" (1.5 T) conditions. Note that with the field "on", the drift time contours, Fig. 13, are almost circular except near the corners of the drift cell where some crossing of charge from one cell to another occurs. This "crosstalk" between drift cells 
Table 4

Capacitance of the wire chambers

\begin{tabular}{llll}
\hline Layer & \multicolumn{3}{l}{ Capacitance $[\mathrm{pf} / \mathrm{m}]$} \\
\cline { 2 - 4 } & MWPC $^{\text {a }}$ & DC1 & DC2 \\
\hline 1 & 10.8 & 9.0 & 8.6 \\
2 & 10.8 & 9.0 & 8.5 \\
3 & 10.8 & 9.0 & 8.4 \\
\hline
\end{tabular}

${ }^{a}$ Determined for a planar chamber, see ref. [26].

can present a problem for various trajectories. One notes that in most, but not all, cases the crosstalk comes at large drift times and can be removed by a time cut.

\section{Gas}

The MWPC was operated with a special gas mixture. It was desired to have high efficiency, fast drift speed, and stability over a wide operating voltage. The basic gas, argone-isobutane, is modified by the addition of methane to increase drift speed. Fig. 17 shows the voltage plateau for various percentages of methane. As methane is added the drift speed increases, but stability decreases. A compromise was chosen at the gas mixture of $20 \%$ isobutane, $58 \%$ methane, and $22 \%$ argon. This gas has about a $40 \%$ increase in drift velocity over argon/ethane, and a voltage plateau of about $150 \mathrm{~V}$.

Because of the unusal spacing of the MWPC it was found that a fast gas mixture involving $\mathrm{CF}_{4}$ was not suitable. For the same reasons magic gas was not expected to work satisfactorily, although it was not tried. The drift chambers were operated with a standard mixture of argon/ ethane.

\section{Scintillators}

The scintillators for the detector are made from Bicron BC412 [23]. Each scintillator bar is approximately $5 \mathrm{~cm}$ wide, $180 \mathrm{~cm}$ long, and $1 \mathrm{~cm}$ thick. The sides of the scintillators are tapered so that they form a barrel when assembled. The $5 \mathrm{~cm} \times 180 \mathrm{~cm}$ surfaces have a cast finish, the ends are sanded and polished, and the sides have a diamond m.11 finish. Bench tests demonstrated that the light attenuation along the bars was comparable for either diamond mill or sanded and polished sides. The support structures for the scintillators in layers 2 and 3 are the outside Al support CAN for the wire chambers of layers 1 and 2 respectively. A separate $\mathrm{Al}$ support cylinder was constructed to hold the layer 1 scintillator barrel. A layer of foam [14] is added to the Al support barrel and ground to the proper diameter, and then black felt is put on top of the foam in order to protect the scintillator surface and to aid in light sealing. Each scintillator is wrapped with $25 \mu \mathrm{m}$ singly aluminized mylar before it is placed on the barrel. Kapton tape is wrapped around the entire barrel to compress the scintillators against the felt to hold them in place. A layer of $50 \mu \mathrm{m}$ doubly aluminized mylar is then wrapped around the entire assembly to assist in light sealing.

Light from each end of the scintillator bars is coupled to a Hamamatsu R1356 phototube [23] by a fiber optic light guide that is approximately $3 \mathrm{~m}$ long. The light guides are fabricated from $2 \mathrm{~mm}$ diameter Mitsubishi ED80 optical fiber [23]. Each light guide consists of 109 fibers. End couplers for the light guides are fabricated by injection molding using black nylon. The end coupler that connects to the scintillator is a rectangular shape to match the bar, and it bolts directly onto the $\mathrm{Al}$ support ring for layer 1 and the end caps of next inner spectrometer for layer 2 and 3 . The connector at the phototube end is round and bolts onto the magnetic shield support discussed below. Details of the construction and performance of the light guides can be found in ref. [23].

The photomultiplier tubes are located outside of the superconducting magnet but are still in a fringe field of several hundred Gauss. The mounting system for the phototubes must therefore act also as a magnetic shield. The phototube magnetic shield is assembled by tack-welding 5.75 "soft-steel" tubes with an outside diameter of $5.7 \mathrm{~cm}$

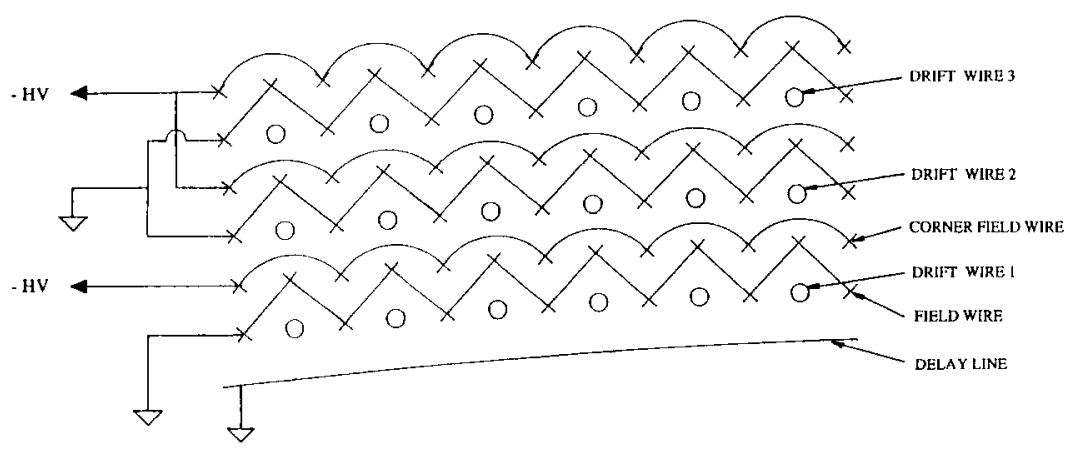

Fig. 15. HV Electrical connections for the drift chamber wire grid. The drift wıre operated at positive HV. 


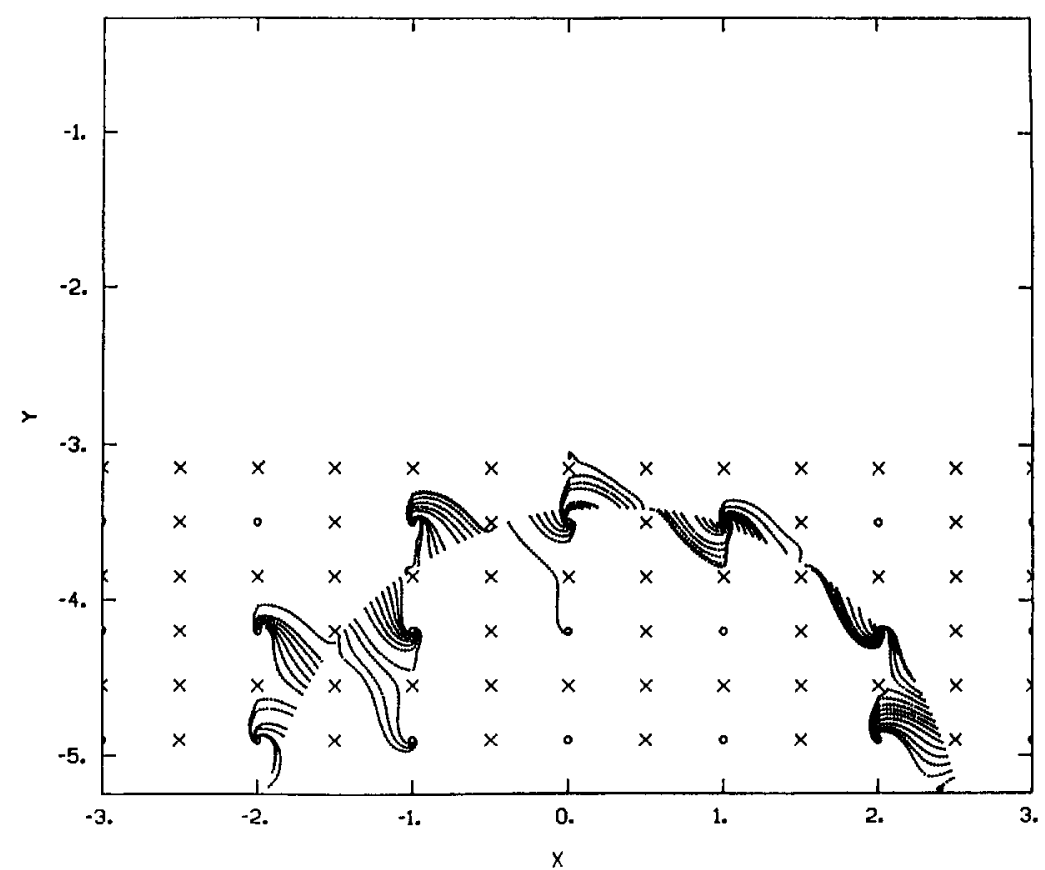

Fig. 16. A typical lepton trajectory through the drift chambers which shows drift paths for the ionization electrons. Note the magnetic field causes a portion of charge to drift into an adjacent drift cell in certain instances. An axial magnetic field of $1.5 \mathrm{~T}$ was applied.

and a wall thickness of $0.95 \mathrm{~cm}$ in a close packed array of T-beams that are bent to match the outside curvature of the magnet. The shield is attached to the magnet by bolting onto pads located at each end of the solenoid. The steel tubes are drilled and tapped on each end to support the phototube base and the light guide coupler. Flat rubber gaskets provide the light seals at each end of the tubes. They also provide electrical insulation to separate the

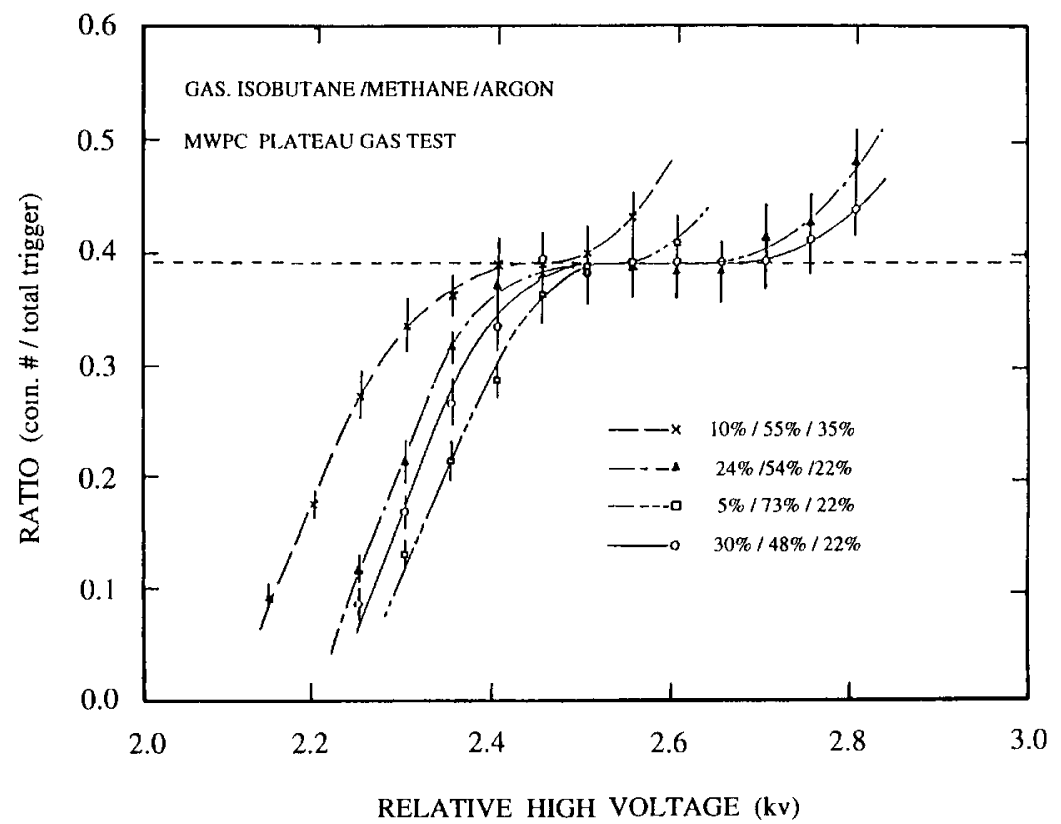

Fig. 17. Relative plateau curves for the MWPC using various gas mixtures of isobutane/methane/argon. 
"dirty" ground of the magnet yoke from the "clean" ground of the phototube bases. The typical magnetic field in the region where the magnetic shield arrays are mounted is $500 \mathrm{G}$, oriented approximately perpendicular to the "soft steel tubes". The "soft-steel" reduces this field to a few Gauss. A standard $1 \mathrm{~mm}$ thick $\mu$-metal shield is then sufficient to reduce the field to well below $1 \mathrm{G}$ inside the combined shields. Checks with a ${ }^{207} \mathrm{Bi}$ source demonstrated that the tube gains shift less than $5 \%$ as the superconducting solenoid field is brought from zero to $16.5 \mathrm{kG}$.

\section{High voltage and electronic support board}

The high voltage and preamp boards for the MWPCs and drift chambers are mounted on a support backplane attached to the end caps. These boards, shaped in arcs, distributed both the low and high voltage power, and the chamber signals to the preamplifier cards. They also contain noise isolation components for the low voltage. The preamp readout was grouped in units of 16 and is connected to the preamplifier boards via a DIN [24] connector on this backplane.

High voltage isolation on the backplane is obtained by placing the high voltage and low voltage on two different boards. Each signal passes through a blocking capacitor from the high voltage to the low voltage board. Signals from the wires were combined into units of 16 and attached to a connector which was inserted into the high voltage board of the back plane. The differential delay line signals are attached directly to the low voltage board since the delay lines were operated at ground potential. The high voltage board has four separate high voltage lines which can be independently connected to each group of 16 sense wires by jumper pins. This allows the MWPC and drift chambers to be operated at different high voltages, and in principle, to operate portions of a given wire chamber at different voltages, although it was found that the latter flexibility was not needed.

\section{Summary}

This paper describes the techniques developed for the mechanical construction of a set of large cylindrical pair spectrometers. These spectrometers were composed of wire chambers and carbon composite cylinders, so that the density of matter through which the conversion pair travels is minimized. The overall apparatus maintains good tolerance levels on dimensions for reliable operation of the detector and the measurment of the kinematic parameters. Future papers will describe the readout electronics and the operating characteristics of the detector.
Appendix A. Stress deflection for a right circular cylinder

\section{A.1. Introduction}

This is a calculation of the stress induced deviations from roundness of a right circular cylinder such as those used in the MEGA photon arm drift chambers. The calculation is based on differential equations which relate the deflections from roundness to stresses on the cylinder. These stresses are represented by external pressures on the cylinder surface.

The mathematical model used in this calculation is commonly referred to as the membrane model for surfaces of revolution. A thorough treatment of the model can be found in ref. [25]. Our notation is identical to that reference.

\section{A.2. The membrane model}

The following parameters will be used:

$a=$ radius of the cylinder,

$h=$ half thickness of the cylinder wall,

$L=$ length of the cylinder,

$\rho=$ density of the material,

$u=$ Poissons ratio for the material,

$E=$ Young's modulus for the material.

The cylinder coordinates are:

$l=$ longitudinal coordinate,

$\psi=$ angular coordinate.

Also, the following functions will be used:

$u(l, \psi)=$ displacement of element in the radial direction,

$s(l, \psi)=$ displacement of element in the $\psi$ direction.

$v(l, \psi)=$ adisplacement of element in the longitudinal direction,

$P_{\mathrm{r}}(l, \psi)=$ external pressure in the radial direction,

$P_{\psi}(l, \psi)=$ external pressure in the $\psi$ direction,

$P_{1}(l, \psi)=$ external pressure in the longitundinal direction.

The coordinate $l$ is taken to be 0 on one end, $L$ on the other end. $\psi=0$ is at the bottom of the (horizontally positioned) cylinder with $\pi$ or $-\pi$ at the top. Looking down the $l=0$ side, $\psi$ increases in the counterclockwise direction.

The stress displacements $u, s$, and $v$ at every point $(a, l, \psi)$ are defined by the following map:

As for the external pressures $p_{1}, p_{\mathrm{r}}(l, \psi)$ is defined to be positive for pressures pushing inward, $p_{1}(l, \psi)$ for pressures which push in the positive $l$ direction, and $p_{\psi}(l, \psi)$ for pressures which cause a twist in the counterclockwise direction when looking down the cylinder from the $l=0$ end. 
The differential equations relating the deflections to the external pressures are as follows:

$$
\begin{aligned}
& \frac{1}{a} \frac{\partial u}{\partial \psi}+\frac{1}{a} \frac{\partial^{2} s}{\partial \psi^{2}}+\frac{a(1-\nu)}{2} \frac{\partial^{2} s}{\partial l^{2}}+\frac{1+\nu}{2} \frac{\partial^{2} v}{\partial \psi \partial l} \\
& -\frac{h^{2}}{3 a^{3}} \frac{\partial^{3} u}{\partial \psi^{3}}-\frac{h^{2}}{3 a} \frac{\partial^{3} u}{\partial \psi \partial l^{2}}=-p_{\psi} \frac{a\left(1-\nu^{2}\right)}{2 E h}, \\
& a \frac{\partial^{2} v}{\partial l^{2}}+\frac{1-\nu}{2 a} \frac{\partial^{2} v}{\partial \psi^{2}}+\nu \frac{\partial u}{\partial l}+\frac{1+\nu}{2} \frac{\partial^{2} s}{\partial \psi \partial l} \\
& =-p_{l} \frac{a\left(1-\nu^{2}\right)}{2 E h},
\end{aligned}
$$

where

$\nabla^{4}=\frac{\partial^{4}}{\partial l^{4}}+\frac{2}{a^{2}} \frac{\partial^{4}}{\partial \psi^{2} \partial l^{2}}+\frac{1}{a^{4}} \frac{\partial^{4}}{\partial \psi^{4}}$.

By inspection of Eqs. (2), (3), and (4), we see that rescaling the external pressures by a constant factor $\alpha$, that is:

$\left(p_{\mathrm{r}}, p_{\psi}, p_{1}\right) \rightarrow\left(\alpha p_{\mathrm{r}}, \alpha p_{\psi}, \alpha p_{1}\right)$

will result in a rescaling of the deflections by the same factor, $(u, s, v) \rightarrow(\alpha u, \alpha s, \alpha v)$. Therefore the "shape" of any set of deflections is independent of the overall normalization of the external pressures.

The material is completely characterized by the parameters $E, \rho$, and $v$. In this model, the cylinder material is assumed to be uniform. The fact that the cylinder is comprised of several different types of materials is taken into account by using a weighted average of each quantity over the density and Young's modulus. That is

$E=\frac{1}{2 h} \sum_{\text {Ldyers }} t_{j} E_{f}$,

and

$\rho=\frac{1}{2 h} \sum_{\text {Layers }} t_{j} \rho_{j}$.

$t$, and $\rho_{J}$ are the thickness and density for layer $j$ respectively, and $2 h$ is the total thickness.

As for $v$, Poisson's ratio does not vary much for a wide range of materials. We will take a typical value of $v=0.3$ in the following.

The values used for all the parameters involved in these calculations are given in Table 5 .

\section{A.3. The gravitational deflection}

When positioned horizontally, there will be a tendency for the cylinders to sag from their own weight. This could be a problem, in particular for the cylinders containing a layer of lead. The greatest deflection will of course be at
Table 5

Parameter values

$a=13.19$ in.

$L=70.87$ in.

$2 h=0.098$ in.

$t_{\mathrm{pb}}=0.01 \mathrm{in}$.

$t_{\mathrm{a} 1}=0.004 \mathrm{in}$.

$t_{\mathrm{cf}}=0.038 \mathrm{in}$. (or 0$)$

$t_{\mathrm{roh}}=0.084 \mathrm{in.}-t_{\mathrm{cf}}$

$E_{\mathrm{ph}}=2.1 \times 10^{6} \mathrm{lbs} . /$ in $^{2}$

$E_{\mathrm{dl}}=9.7 \times 10^{6} \mathrm{lbs} . / \mathrm{in}^{2}$

$E_{\mathrm{cf}}=1.4 \times 10^{6} \mathrm{lbs} . /$ in. $^{2}$

$E_{\mathrm{roh}}=2.5 \times 10^{4} \mathrm{lbs} . /$ in. $^{2}$

$\rho_{\mathrm{pb}}=0.397 \mathrm{lbs} . /$ in. $^{3}$

$\rho_{\mathrm{al}}=0.098 \mathrm{lbs} . / \mathrm{in}^{3}$

$\rho_{\mathrm{cf}}=0.056 \mathrm{lbs} . / \mathrm{in.}^{3}$

$\rho_{\text {roh }}=0.004 \mathrm{lbs} . /$ in. $^{3}$

$\nu=0.3$

$(l=L / 2, \psi=0)$ and $(l=L / 2, \psi=\pi)$ We show how the complete expressions $u(l, \psi), v(l, \psi)$, and $s(l, \psi)$ can be found.

The gravitational force on a unit area element of the cylinder is given by (directed downward):

$p_{\text {grav }}=2 \rho h Q(l)$,

where $Q(1)$ is a function which mimics the fact that the cylinder is epoxied into a groove $\delta=\frac{1}{4}$ in. deep on each end. The deflection pressure on this part of the cylinder should therefore be 0 . So $Q$ should be of the form:

$Q(l)=\left\{\begin{array}{lll}0 & \text { if } & 0 \leq l \leq \delta ; \\ 1 & \text { if } \quad \delta<l<L-\delta ; \\ 0 & \text { if } \quad L-\delta \leq l \leq L .\end{array}\right.$

The gravitational pressure will yield a non-zero value for the pressures $p_{\mathrm{r}}(l, \psi)$ and $p_{\psi}(l, \psi)$. They are:

$p_{\mathrm{r}}(l, \psi)=-2 h \rho \cos (\psi) Q(l)$,

$p_{\psi}(l, \psi)=-2 h \rho \sin (\psi) Q(l)$.

To solve this system of linear partial differential equations, we can assume that the deflections are products of harmonic functions in $l$ and $\psi$. Boundary conditions and symmetry arguments will restrict the form of these products to the following combinations:

$$
\begin{aligned}
& u(l, \psi)=\sum_{n=0}^{\infty} \sum_{m=1}^{\infty} h A_{m, n} \cos (n \psi) \sin \left(\frac{m \pi l}{L}\right), \\
& s(l, \psi)=\sum_{n=0}^{\infty} \sum_{m=1}^{\infty} h B_{m, n} \sin (n \psi) \sin \left(\frac{m \pi l}{L}\right), \\
& v(l, \psi)=\sum_{n=0}^{\infty} \sum_{m=1}^{\infty} h C_{m, n} \cos (n \psi) \cos \left(\frac{m \pi l}{L}\right) .
\end{aligned}
$$

As an example, consider $u$. It must be 0 at $l=0$ and $l=L$. This forces it to have the $\sin (m \pi l / L)$ behavior. 
Also, it should be symmetric under $\psi \rightarrow-\psi$, and this restricts it to the $\cos (n \psi)$ form. Similar arguments can be made for the quantities $s(l, \psi)$ and $v(l, \psi)$.

We can also decompose $Q(l)$ along the interval $[0, L]$ :

$$
\begin{aligned}
Q(l)= & \sum_{m=0}^{\infty} \frac{4}{(2 m+1) \pi} \cos \left(\frac{(2 m+1) \pi \delta}{L}\right) \\
& \times \sin \left(\frac{(2 m+1) \pi l}{L}\right) .
\end{aligned}
$$

We can then substitute the expansions for the deflections and $Q(l)$ into Eqs. (2), (3), and (4). Coefficients of the independent harmonic functions are equated on each side to obtain equations for the $A_{i, j}$ 's, $B_{i, j}$ 's and the $C_{i, j}$ 's in terms of known quantities. The only nonzero terms found are of the type $A_{2 m+1,1}, B_{2 m+1,1}$, and $C_{2 m+1,1}$. This can be understood quite easily if one examines the expressions for $p_{\mathrm{r}}(l, \psi)$ and $p_{\psi}(l, \psi)$ given above.

In our application the cylinders are very thin $(2 h=$ $2 \mathrm{~mm}$ ), therefore the terms in Eqs. (2) and (3) involving $h^{2}$ can be dropped. This can be understood physically by realizing that the gravitational force on an element of the cylinder and the structural strength of the same element against distortion are both proportional to $h$. When these terms are ignored the expansion coefficients are found to be:

$$
\begin{aligned}
A_{2 m+1,1}= & \frac{4 \rho a^{2}}{(2 m+1) \pi h E} \cos \left(\frac{(2 m+1) \pi \delta}{L}\right) \\
& \times\left(\frac{2}{\kappa_{2 m+1}^{4}}+\frac{\nu+4}{\kappa_{2 m+1}^{2}}+1\right), \\
B_{2 m+1,1}= & \frac{-4 \rho a^{2}}{(2 m+1) \pi h E} \cos \left(\frac{(2 m+1) \pi \delta}{L}\right) \\
& \times\left(\frac{2}{\kappa_{2 m+1}^{4}}+\frac{3 \nu+4}{\kappa_{2 m+1}^{2}}\right), \\
C_{2 m+1,1}= & \frac{4 \rho a^{2}}{(2 m+1) \pi h E} \cos \left(\frac{(2 m+1) \pi \delta}{L}\right) \\
& \times\left(\frac{\nu}{\kappa_{2 m+1}}-\frac{2}{\kappa_{2 m+1}^{3}}\right) .
\end{aligned}
$$

Here $\kappa_{m}=(m \pi a) / L$.

Putting these back into Eqs. (5), (6), and (7) gives the complete expressions for $u(l, \psi), v(l, \psi)$, and $s(l, \psi)$.

In this calculation, the interesting quantity is $u(L / 2,0)$. It is found that the series for $u(l, \psi)$ converges very rapidly. The $m=1$ term in Eq. (5) yields a result that is within a few $\%$ of the entire series at $l=L / 2$, that is:

$$
u\left(\frac{L}{2}, 0\right) \approx h A_{1,1} \text {. }
$$

It is interesting to examine Eq. (12) in the large $L$, fixed a limit. The leading term goes like $\kappa_{1}^{-4} \sim L^{4} / a^{4}$ and is independent of $v$. (Actually $A_{l, j}, B_{l, j}$ and $C_{l, j}$ are all independent of $v$ in this limit.) If we set $\delta=0$ we obtain for the maximum radial deflection:

$u\left(\frac{L}{2}, 0\right) \rightarrow \frac{8}{\pi^{5}} \frac{\rho L^{4}}{E a^{2}} \approx \frac{5}{384} \frac{W L^{3}}{E I}$.

This last expression has been written in terms of the weight $W=4 \pi a L h \rho$ and the moment for a thin walled cylinder of radius $a$ and wall thickness $2 h, I=2 \pi a^{3} h$.

This formula should be compared to the maximum deflection of a beam of moment $I$, Young's modulus $E$, and weight $W$ in the fixed end and free end cases. They are:

$\begin{aligned} \operatorname{Def}_{\text {free }}^{\text {beam }} & =\frac{5}{384} \frac{W L^{3}}{E I}, \\ \operatorname{Def}_{\text {fixed }}^{\text {beam }} & =\frac{1}{384} \frac{W L^{3}}{E I} .\end{aligned}$

Our result reduces to the free end case in the large $L$ limit, as one expects. However, it should be pointed out that Eq. (13) is not a good approximation for calculating the deflections of the cylinders for the MEGA photon are chambers. MEGA has $L=180 \mathrm{~cm}$ and the smallest radius cylinder has $a=33.6 \mathrm{~cm}$. Using these dimensions for the cylinder will underestimate the maximum deflection by a factor of $\frac{1}{2}$. When the full expression for $A_{1,1}$ is used, we obtain a maximum deflection of $10 \mu$.

\section{A.4. Resistance to local deflections}

We will now ask the model to calculate the deflection due to a pressure exerted on a rectangular area of size $(a \Delta \Psi) \times \Delta L$.

We present the results with just enough detail so that the interested reader can check the calculation. Obviously the only nonzero external force is $p_{\mathrm{T}}(l, \Psi)$. This takes the form:

$p_{\mathrm{r}}(l, \psi)=\frac{F}{a \Delta \psi \Delta L} Q(l, \psi)=p_{\mathrm{r}}^{0} Q(l, \psi)$.

where $F$ is the total force applied and $Q$ represents a " unit bump" centered at the point ( $l=L / 2, \Psi=0)$, that is:

$Q(l, \psi)=\left\{\begin{array}{cc}1 \quad & \text { if }(l, \psi) \in\left[-\frac{\Delta L}{2}, \frac{\Delta L}{2}\right] \\ & \times\left[-\frac{\Delta \psi}{2}, \frac{\Delta \psi}{2}\right] \\ 0 & \text { otherwise. }\end{array}\right.$

Putting the pressure point at $(l=L / 2, \Psi=0)$ gives the quantities $u(l, \Psi), v(l, \Psi)$, and $s(l, \Psi)$ the same symmetries as in the gravitational sag problem, so the expan- 
sions (5), (6) and (7) are still valid. As for $Q(l, \Psi)$ without too much work its expansion is found to be:

$$
\begin{aligned}
Q(l, \psi)= & \sum_{n=0}^{\infty} \sum_{m=0}^{\infty} b_{2 m+1, n} \cos (n \psi) \\
& \times \sin \left(\frac{(2 m+1) \pi l}{L}\right) .
\end{aligned}
$$

The coefficients $b_{2 m+1,1}$ are:

$$
\begin{aligned}
b_{2 m+1,0}= & \frac{2 \Delta \psi(-1)^{m}}{(2 m+1) \pi^{2}} \sin \left(\frac{(2 m+1) \pi \Delta L}{2 L}\right), \\
b_{2 m+1, n>0}= & \frac{8(-1)^{m}}{(2 m+1) n \pi^{2}} \sin \left(\frac{n \Delta \psi}{2}\right) \\
& \times \sin \left(\frac{(2 m+1) \pi \Delta L}{2 L}\right) .
\end{aligned}
$$

It's fairly easy to see that if $\Delta \Psi=2 \pi$ and $\Delta L=L-$ $2 \delta$, we recover $Q(l)$ from the previous calculation.

Substitution is again performed, however it is found in this problem that the $\mathrm{O}\left(h^{2}\right)$ terms in the differential equations cannot be ignored. This is because although the material strength is proportional to $h$, the force in this problem is not. This is expressed mathematically by the fact that if the $\mathrm{O}\left(h^{2}\right)$ terms are dropped, the series in Eq. (5) will not converge in the $\cos (n \Psi)$ sum. The coefficient equations are the following:

$$
\begin{aligned}
& A_{2 m+1, n}\left(1+\frac{\epsilon^{2}}{3}\left(n^{2}+\kappa_{2 m+1}^{2}\right)^{2}\right)+n B_{2 m+1, n} \\
& -\nu \kappa_{2 m+1} C_{2 m+1, n}=-\gamma b_{2 m+1, n}, \\
& A_{2 m+1, n}\left(n+\frac{\epsilon^{2}}{3}\left(n^{3}+n \kappa_{2 m+1}^{2}\right)\right) \\
& +B_{2 m+1, n}\left(n^{2}+\frac{1-\nu}{2} \kappa_{2 m+1}^{2}\right) \\
& \quad-\frac{1+\nu}{2} n \kappa_{2 m+1} C_{2 m+1, n}=0, \\
& \nu \kappa_{2 m+1} A_{2 m+1, n}+\frac{1+\nu}{2} n \kappa_{2 m+1} B_{2 m+1, n} \\
& -C_{2 m+1, n}\left(\kappa_{2 m+1} u 2+\frac{1-\nu}{2} n^{2}\right)=0 .
\end{aligned}
$$

Here $\epsilon=h / a$ and $\gamma=p_{\mathrm{r}}^{0}\left(1-v^{2}\right) /\left(2 \epsilon^{2} E\right)$.

These equations are presented for two reasons. The first reason is so that anyone interested can solve them and check the final result. The other reason is to be able to check the gravitational sag problem. If we set $\epsilon=0$, shift $b_{2 m+1, n} \rightarrow b_{2 m+1, n-1}$, set $n=1$, use $b_{2 m+1, n}(\Delta \Psi=$ $2 \pi, \Delta L=L-2 \delta$ ), set the second equation equal to $-\gamma b_{2 m+1, n-1}$ (instead of equal to 0 ), and redefine $p_{\mathrm{r}}^{0}$ to be $2 h \rho$, we get the equations for the coefficients of the gravitational sag problem.
In order to plot the shape for some applied force, we must go back to the definition of the deflections given by Eq. (1). We wish to look at constant $l$ and constant $\Psi$ cross sections of the cylinder with the pressure applied. The most interesting places to look are at $l=L / 2$ and at $\Psi=0$. These cross sections are also easy to compute because by symmetry, $s(l, 0)=0$ and $v(L / 2, \Psi)=0$, simplifying the implementation of Eq. (1) enormously.

Plots of these two views are given in Figs. 18 and 19. The applied pressure is from a force of $F=7.1 \mathrm{~N}$, applied on an area which is $1.3 \times 1.3 \mathrm{~cm}^{2}$. Although the shape of the dent is dependent on the applied force, the maximum deflection is proportional to $p_{\mathrm{r}}^{0} / E$, so the maximum deflection for any material with any force (and the same wall thickness) is easily obtained from this result.

\section{A.5. Cylinder compression}

As a final study, we examine the deflection of a cylinder when a force is applied in the longitundinal direction, for example if the cylinders would be required to support the wire tension. In this case, the only nonzero external pressure will be $p_{1}$. We have azimuthal symmetry, so all the deflections are functions of $l$ only. Also from this symmetry, we must have $s(l) \equiv 0$. The differential equations then reduce to:

$$
\begin{aligned}
& \frac{a h^{2}}{3} \frac{\mathrm{d}^{4} u(l)}{\mathrm{d} l^{4}}+\frac{1}{a} u(l)+\nu \frac{\mathrm{d} v(l)}{\mathrm{d} l}=0, \\
& a \frac{\mathrm{d}^{2} v(l)}{\mathrm{d} l^{2}}+\nu \frac{\mathrm{d} u(l)}{\mathrm{d} l}=-\frac{a\left(1-\nu^{2}\right)}{2 E h} p_{l}(l) .
\end{aligned}
$$

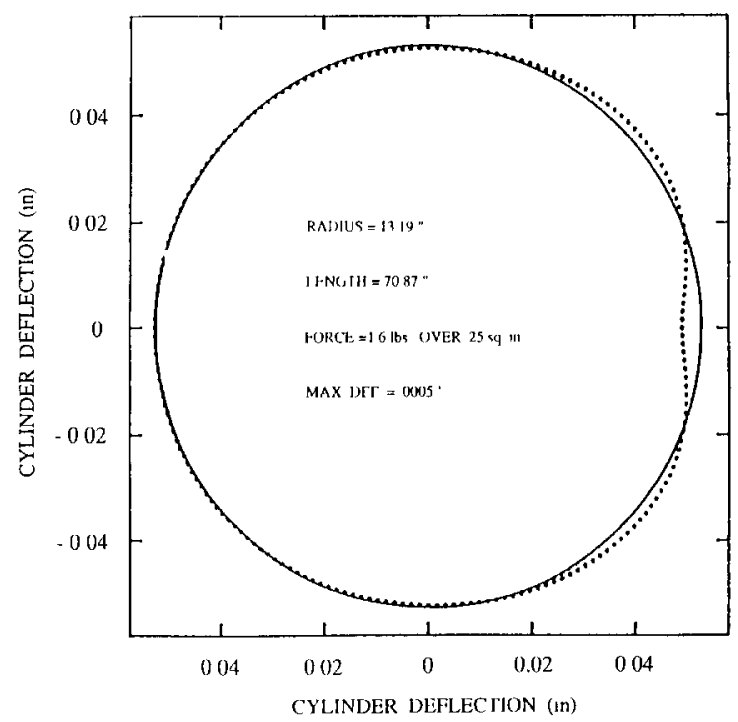

Fig. 18. Deflection of a $1 \mathrm{~mm}$ carbon fiber cylinder about a circumference at mid-length under the conditions shown and described in the text 
These can be solved iteratively for $u(l)$ in terms of the function $p_{1}(l)$. To do this, one first integrates Eq. (15) form 0 to $l$ to obtain:

$\frac{\mathrm{d} v(l)}{\mathrm{d} l}=-\frac{1-\nu^{2}}{2 E h} \int_{0}^{l} p_{l}\left(l^{\prime}\right) \mathrm{d} l^{\prime}-\frac{\nu}{a}(l)$.

Substituting Eq. (16) into Eq. (14) and writing things in terms of the dimensionless quantities $\bar{u}=u / a$ and $\bar{l}=l / a$, the expansion parameter $\epsilon=h / a$ and $p_{1}(l)=p_{1}(l)$ gives:

$$
\frac{\epsilon^{2}}{3} \frac{\mathrm{d}^{4} \bar{u}}{\mathrm{~d} \bar{l}^{4}}+\left(1-\nu^{2}\right) \bar{u}=\frac{\nu\left(1-\nu^{2}\right) a}{2 E h} \int_{0}^{\bar{l}} \bar{p}_{l}\left(\bar{l}^{\prime}\right) \mathrm{d} \bar{l}^{\prime} .
$$

The function $\bar{u}$ is then expanded in powers of $\epsilon^{2}$, that is: $\bar{u}(\bar{l})=\sum_{i=0}^{\infty} \epsilon^{2 i} \bar{u}_{i}(\bar{l})$.

Putting this expansion into Eq. (17) and equating powers of $\epsilon^{2}$ fixes $\bar{u}_{0}$ gives a recurrence relation between $\mathrm{d}^{4} \bar{u}_{i} / \mathrm{d} \bar{l}^{4}$ and $\bar{u}_{i+1}$. The expansion is found to be:

$$
\begin{aligned}
\bar{u}(\bar{l})= & \frac{a v}{2 E h}\left(\int_{0}^{\bar{l}} \bar{p}_{l}\left(\bar{l}^{\prime}\right) \mathrm{d} \bar{l}^{\prime}+\sum_{t=0}^{\infty}\left[\frac{\epsilon^{2}}{3\left(1-\nu^{2}\right)}\right]^{\imath+1}\right. \\
& \left.\times \frac{\mathrm{d}^{3+4 t}}{\mathrm{~d} \bar{l}^{3+4} t} \bar{p}_{l}(\bar{l})\right)
\end{aligned}
$$

In the 1988 drift chamber prototype the cylinders were fabricated with rohacell and no carbon fiber. The innermost and outermost layers were aluminized kapton. The

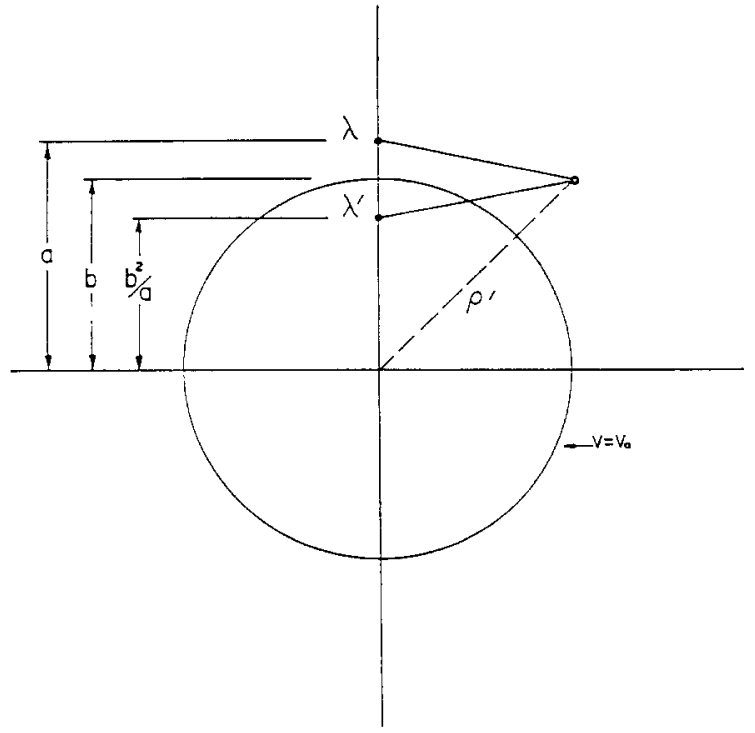

Fig. 20. The figure used to obtain the Green Function for a wire above a conducting cylinder.

cylinders were pressed longitudinally from both sides by a force which was equal to the total wire tension. We will denote this force by $F$ and assume it was distributed down the cyliner wall in some smooth way by the outer and inner kapton layers. We should model this force with some assumption on the form of $p_{1}(l)$. It is expected that $p_{1}$ should be decreasing for increasing $l$ and at some $l=l_{0}$

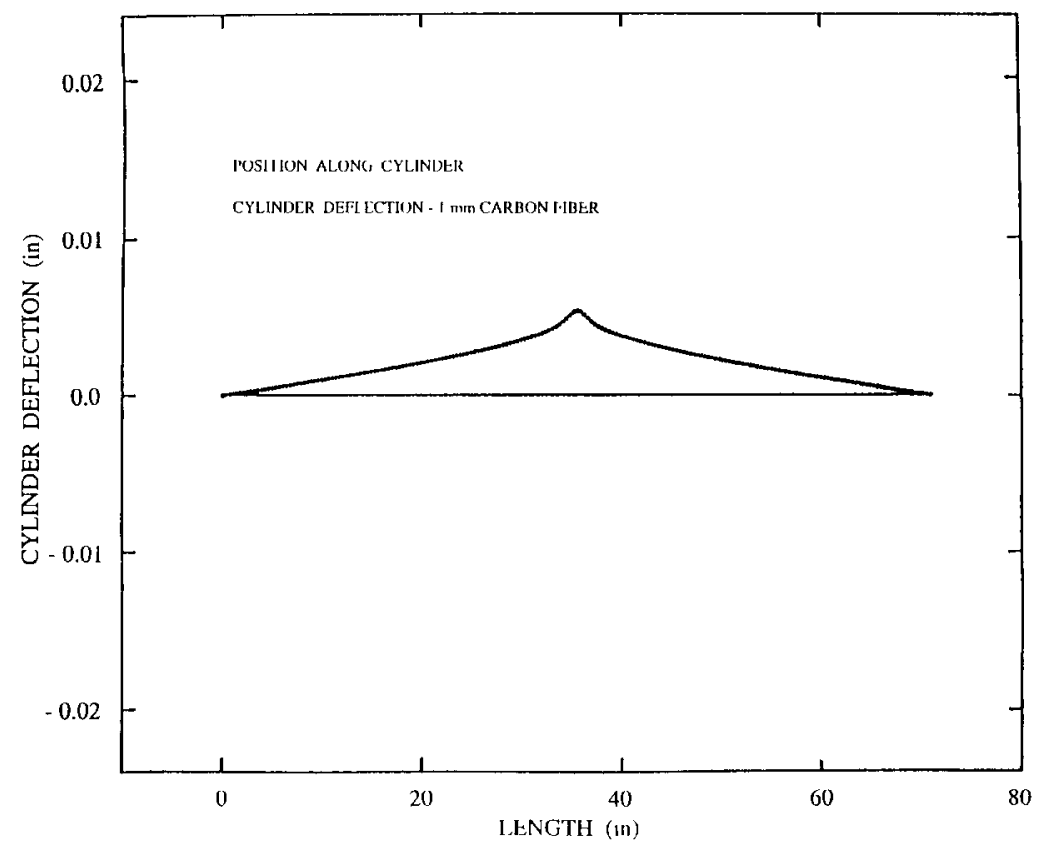

Fig. 19. Axial deflection of a $1 \mathrm{~mm}$ carbon fiber cylinder under the conditıons described in the text and Fig. A-1 
( $l_{0}=L / 2$ if the cylinders were fabricated correctly), $p_{1}\left(l_{0}\right)=0 . \quad p_{1}$ will then go negative due to the force pushing from the other side. It should be obvious that:

$F=2 \pi a \int_{0}^{l_{n}} p_{l}\left(l^{\prime}\right) \mathrm{d} l^{\prime}=-2 \pi a \int_{l_{0}}^{L} p_{l}\left(l^{\prime}\right) \mathrm{d} l^{\prime}$.

The point is that if $p_{1}$ is smooth enough, we can set $\epsilon^{2}=0$ in the expansion for $u(l)\left(\epsilon^{2} / 3\left(1-v^{2}\right)=5.5 \times\right.$ $10^{-6}$ for our dimensions). The maximum deflection will be independent of the exact form of $p_{1}$ and will be $u\left(l_{0}\right)$ and so we obtain, finally:

$u_{\max }=\frac{F a \nu}{4 \pi E h}$.

This result is independent of the form of $p_{1}$ and has been checked for a variety of functional forms of $p_{1}$. In fact, it was discovered numerically, which motivated the above derivation.

\section{Appendix B. Capacitance of a cylindrical wire grid above a cylindrical conducting plane}

The potential of a thin wire above an infinite, cylindrical conducting plane may be obtained from the Green's Function. By reference to Fig. 20, this function in cylindrical coordinates

$G\left(\rho, \rho^{\prime}\right)=\ln \left[\frac{\left(\rho^{2}-b^{2}\right)\left(\rho^{\prime 2}-b^{2}\right)}{b^{2}\left(\rho-\rho^{\prime}\right)^{2}}+1\right]$

Note the symmetry in the exchange of $\rho$ and $\rho^{\prime}$, and the fact that $G=0$ when $\rho=b$, i.e. on the surface of the conducting plane. Thus the potential is:

$V(\rho)=\int \mathrm{d} s^{\prime} \sigma G-\frac{1}{4 \pi} V_{0} \int_{\rho^{\prime}=b} \mathrm{~d} l^{\prime}(\nabla G \cdot \boldsymbol{n})$

where it is assumed that the surface charge, $\sigma$, is the charge on each wire, and $\boldsymbol{n}$ is the unit vector perpendicular to the surface of the wire. For a grid of $N$ very thin wires placed at radius, $a$, and spaced every $2 \phi$ rad. apart, the surface charge is then:

$\sigma=\frac{-\lambda}{2 \epsilon_{0}} \sum_{m=0}^{N} \frac{\delta\left(\rho^{\prime}-a\right)}{\rho^{\prime}} \delta\left(\phi^{\prime}-2 m \phi_{0}\right)$.

Here $\lambda$ is the linear charge density (charge per unit length) on a wire of the grid. The gradient term is therefore:

$$
\begin{aligned}
& (V G \cdot \hat{\boldsymbol{n}}) \underset{\rho^{\prime}=b}{ } \\
& =\frac{-4 \rho\left[b \rho\left(1+\cos ^{2} \phi\right)-\left(b^{2}+\rho^{2}\right) \cos \phi\right]}{\left(\rho^{2}+b^{2}-2 \rho b \cos \phi\right)^{2}}
\end{aligned}
$$

Substitution into Eq. (B-2) and completing the integral over the $\delta$ functions gives the potential:

$$
\begin{aligned}
V= & \frac{\lambda}{4 \pi \epsilon_{0}} \sum_{m=0}^{N} \\
& \times \ln \left[\frac{\left(\rho^{2}-b^{2}\right)\left(a^{2}-b^{2}\right)}{b\left\{\rho^{2}+a^{2}-2 \rho a \cos \left(\phi-2 m \phi_{0}\right)\right\}}+1\right]+\frac{2 b \rho}{\pi} \\
& \times V_{0} \int_{0}^{2 \pi} \mathrm{d} \phi\left[\frac{b \rho\left(1+\cos ^{2} \phi\right)-\left(b^{2}+\rho^{2}\right) \cos \phi}{\left(\rho^{2}+b^{2}-2 \rho b \cos \phi\right)^{2}}\right] .
\end{aligned}
$$

Now for the MEGA photon detector, we superimpose the above solution to obtain the solution for the geometry shown in Fig. 20. Note that the problem has symmetry under rotation by an angle of $2 \phi_{0}$. Each set of wires can have a different potential. The charge for each wire is calculated by solving the linear array:

$$
\begin{aligned}
V_{j}= & \sum_{i} \frac{r_{1}}{4 \pi \epsilon_{0}} \sum_{m=0}^{N} \\
& \times \ln \left[\frac{\left(r_{l}^{2}-b^{2}\right)\left(r_{i}^{2}-b^{2}\right)}{b^{2}\left\{r_{i}^{2}+r_{j}^{2}-2 r_{1} r_{j} \cos \left(\phi_{j}-\phi_{1}-2 m \phi\right)\right\}}+1\right] .
\end{aligned}
$$

where:

$\phi_{i}= \begin{cases}0 & i \text { odd } \\ \phi_{0} & i \text { even }\end{cases}$

The boundry condition that $V=0$ if $r_{1}=0$ has been applied. Eq. (B-6) has the linear form:

$V_{j}=\sum \frac{\lambda_{\imath}}{4 \pi \epsilon_{0}} A_{\imath \jmath}-B_{J}$

with $B_{J}=0$ for the problem of interest here. Inversion of this equation gives the linear charge densities on the wires:

$\lambda_{t}=4 \pi \epsilon_{0} \sum_{j} \tilde{A_{i j}}\left(V_{j}-B_{j}\right)$.

Finally the capacitance is found from this equation by placing unit potential on the wire of interest, and zero potential on all other conductors. Table 4 gives the capacitance of the various wires of the grid.

\section{References}

[1] R.D. Bolton, et al., Phys. Rev Lett. 53 (1984) 1415.

[2] R.D. Bolton et al, Phys. Rev. Lett 56, (1986) 2461.

[3] LASS Magnet, SLAC-PUB-2642, Oct. 1980

[4] A.R. Erwin et al., Nucl. Inst. and Meth A 237, (1985) 493.

[5] The grinding wheel was $127 \mathrm{~cm} \times 101 \mathrm{~cm}$ with \#40 grit.

[6] R T. Gilman, inc, P.O. Box 470, Grafton, WI 53024-0470. 
[7] Hexcel GA045-12, Hexcel Corp, 555 Republic Dr., Suite 306, Plano, TX 75074.

[8] Hexcel Resin Tape 2410, Hexcel Hardener Type 2183, Hexcel Corp, 20701 Nordhoff St., Chatsworth, CA 91311.

[9] Hexcel Carbon Fiber Tape 282, Hexcel Corp, Plano, TX 75074 .

[10] Lead Sheet $0.26 \mathrm{~cm} \times 55.9 \mathrm{~cm}$, A010-24-CL, Vulcan Lead Products, 1400 W. Pierce St., Milwaukee, WI 53204.

[11] 3M Remount Adhesive, Adhesive Systems, 3M Industrial Specialities Division, St. Paul, MN 55144-1000.

[12] Epoxy type 216, Crest Products 10550 Lawson River Ave., Fountain Valley, CA 92708.

[13] Polyurethane foam, Clark Foam, 25887 Crown Valley Parkway, South Laguna, CA 92677.

[14] John A. Kadyk, Nucl. Inst. and Meth. A 300, (1991) 436.

[15] Aluminum foil $50 \mu \mathrm{m} \times 25.4 \mathrm{~cm}$ special rolled and cut, Hardness 19, Alloy 1145, A J. Oster, 22833 La Palma Ave,, Yorba Linda, CA 92686.

[16] $1 \mathrm{oz}$. Cu on $76 \mu$ Kapton foil with $25 \mu$ Dupont Pyralux Adhesive. Trace w1dth $0.92 \mathrm{~cm}$; Spacing $0076 \mathrm{~cm}$; Repeat $1 \mathrm{~cm}$, Tech-Etch 45 Aldrin Road, Plymouth, MA 02360.
[17] TRA-DUCT 2902, TRA-CON, 55 North St., Medford, MA 02155 .

[18] Sense wire was either $25 \mu \mathrm{m}$ or $35 \mu \mathrm{m}, 6 \%$ Au plated $\mathrm{W}$ with ultra finish, California Fine Wire, 338 S. Forth St., P.O. Box 446, Grover City, CA 93433.

[19] Hartzell Manufacturing, 2516 Wabash Ave, St. Paul, MN 55114.

[20] Copper tubing of $0.1 \mathrm{~cm} \mathrm{OD}$ and either $200 \mu$ or $76 \mu$ ID depending on the wire diameter; Uniform Tubes, Inc., Collegeville, PA 19426. Tubes crimped by Squeeze-Eze Power Pack (SP-OZ), Simonds, Inc., 248 Worcester St., Southbridge, MA 01550

[21] F. Sauli, CERN Report 77-09, 1977.

[22] Glyptal, GC-Thorsen Electronics, Rockford, IL, 61101-1209.

[23] C.A. Gagliard, et. al., Nucl. Instr. and Meth., A273, (1988) 117.

[24] DIN specification 41612.

[25] C.E. Turner, Introduction to Plate and Shell Theory, Longmans, 1965

[26] P.M. Morse and H. Feshbach, Methods of Theoretical Physics Vol. II, McGraw-Hill, 1953) p. 1241. 